TAO, Vol. 17, No. 1, 53-89, March 2006

\title{
Model Sensitivities in Numerical Simulations of the Formation of Typhoon Robyn (1993)
}

\author{
Kevin K. W. Cheung ${ }^{1, *}$ and Russell L. Elsberry ${ }^{2}$
}

(Manuscript received 15 August 2004, in final form 15 December 2004)

\begin{abstract}
The PSU-NCAR mesoscale model MM5 with an 81-km (27-km) resolution coarse (fine) grid is used to simulate the formation of Typhoon Robyn (1993) in the western North Pacific. This study examines the roles of mesoscale convective system (MCS) during Robyn's formation, and also focuses on the sensitivity of the simulations of Robyn's intensification to the choice of cumulus parameterization, planetary boundary layer (PBL) parameterization, and the explicit moisture scheme in the model.

The control experiment with the Betts-Miller cumulus parameterization, the PBL treatment as in the NCEP Medium Range Forecast (MRF) model, and the Reisner explicit moisture scheme with predicted number concentrations of snow, ice, and graupel has the closest intensification profile to the best track. The control experiment can also reproduce quite well the successive MCSs associated with the development of Typhoon Robyn. The control experiment is then validated with aircraft observations taken during the TCM-93 field experiment. Varying the cumulus parameterization scheme has the largest impact on the simulations of the intensification of Robyn. An experiment with a more tropical relaxation reference profile in the Betts-Miller scheme, and two others using the Kain-Fritsch and the Grell schemes all underestimate the intensification of Robyn.

In addition to the MRF PBL scheme, the PBL scheme from the NCEP Eta model, the Burk-Thompson, and the Gayno-Seaman schemes are tested. Little impact on the intensification and track of Typhoon Robyn is found from a change in the PBL treatment. Examination of the convection and cloud water in the PBL of these experiments reveals that the PBL in the MRF scheme is relatively dry due to its active vertical mixing, as found in previous studies such as Braun and Tao (2000).

\footnotetext{
${ }^{1}$ National Science and Technology Center for Disaster Reduction, Taipei, Taiwan, ROC

2 Department of Meteorology, Naval Postgraduate School, Montercy, CA, USA

* Corresponding author address. Dr. Kevin K. W. Cheung, National Science and Technology Center for Disaster Reduction, Taipei, Taiwan, ROC; Email: cheung@ncdr.nat.gov.tw
} 


\begin{abstract}
Although the magnitudes of impacts from different explicit moisture calculations are less than that of the cumulus parameterization, they do affect the intensification of Typhoon Robyn through their interaction with the cumulus parameterization. All three explicit schemes (simple ice calculation, mixed-phase model, and Schultz scheme) underestimate the intensity of Robyn. Examination of the convective rainfall shows that the deviations among these experiments are due to modified convective cloud patterns by the explicit moisture variables.
\end{abstract}

(Key words: Tropiacl cyclone formation, Numerical simulation, Model sensitivity)

\title{
1. INTRODUCTION
}

It is well known that formation of a tropical cyclone (TC) is a multi-scale problem. Every formation requires that large-scale environmental conditions satisfy specific criteria. The classic works of Gray (1968), McBride and Zehr (1981), and McBride (1981) identified the basic favorable environmental conditions for TC formations. Cheung (2004) recently re-examined these criteria for TC formations in the western North Pacific (WNP) during a 12-year period. The distributions of the sea-surface temperature (SST), 200-850-hPa vertical shear of the zonal and meridional winds, 500-700-hPa relative humidity $(\mathrm{RH})$, vertical instability measured by the convective available potential energy (CAPE), and 200-hPa divergence associated with these formation cases were identified. A potential formation area (PFA) was defined as the area in which the environmental parameters possess the most probable values as identified in the distributions from the development samples. Cheung (2004) found that the seasonal variation of PFA is moderately correlated with that of TC activity, which indicates that the seasonal cycle of TC activity is basically regulated by large-scale parameters. However, the interannual variability of TC activity is not well explained by PFA variations.

The hypothesis here is that TC formation is likely also governed by the subsynoptic and mesoscale disturbances present within the PFA. This hypothesis is consistent with that of Ritchie et al. (2003), who examined the merger of mesoscale convective vortices (MCVs) during hurricane formations in the Atlantic. In this merger process, some MCSs could survive under strong vertical wind shear or low SST conditions, and be transformed subsequently into TCs. Therefore, TC activity may be modulated not only by the variability of the large-scale environment, but also by these subsynoptic and mesoscale systems. The relatively low skill of global numerical model predictions of TC formations (see e.g., Cheung and Elsberry 2002) compared with that for predicting TC tracks may then be a reflection of an inadequate ability to predict the precise behavior of mesoscale circulations, and in particular their relationships to synoptic circulations during TC formation. This study takes a numerical modeling and case study approach to enhance our understanding of this kind of relationship.

A large number of numerical modeling studies have been done on various aspects of extratropical MCSs. Although the basic environment in the extratropics is different from that in the tropical marine environment, some results concerning the transformation of mid-level 
MCVs into low-level cyclones might be relevant to understanding tropical cyclogenesis. For example, Chen and Frank (1993) used the Pennsylvania State University (PSU)-National Center for Atmospheric Research (NCAR) MM5 model with a modified hybrid convection parameterization scheme to simulate mesovortex genesis in an analytically specified large-scale environment. Their simulations show that a MCV may be produced by localized warming in a region of locally reduced Rossby radius, which induces convergence and creates rotational momentum via geostrophic adjustment. Rogers and Fritsch (2001) also used the MM5 model to simulate a midlatitude MCV that initiates, within the borders of the midlevel vortex circulation, several successive cycles of convection. After each cycle of convection, both the horizontal size of the cyclonic circulation and the magnitude of the potential vorticity associated with the vortex were simulated to increase. A conceptual model was then presented to explain how convective redevelopment within the saturated environment of a MCV causes low-level heights to fall and cyclonic vorticity to be extended downward to the surface. Similar processes might also apply to TC formations.

In the Bister and Emanuel (1997) idealized simulation with a nonhydrostatic, axisymmetric numerical model, the midlevel vortex develops downwards as the cold core is transformed to a warm-core structure. Evaporation of the rain first cools the lower troposphere and leads to the spin-up of a midlevel vortex. Then both the cold anomaly associated with the cyclone and the relatively humid air associated with the evaporatively cooled mesoscale cyclone are important for the subsequent development of the warm core. The stratiform rain must also continue long enough to drive the midlevel vortex down to the boundary layer.

Davis and Bosart $(2001,2002)$ used the MM5 model to simulate three phases of the transformation of a weak baroclinic disturbance into Hurricane Diana (1984). In the first phase with baroclinic and barotropic development, the low-level circulation is strengthened through the axisymmetrization of adjacent potential vorticity anomalies that are generated by condensational heating and then advected toward the incipient storm. The transformation from coldcore to warm-core vortex also occurs in this development stage. In a sensitivity study (Davis and Bosart 2002), the intensification of Hurricane Diana is examined for various choices of cumulus parameterization, boundary-layer treatment, SST, and grid spacing. The simulations with cumulus parameterization schemes that allow more grid-scale precipitation on the 9-km grid exhibit unrealistic grid-scale overturning and slower intensification. Use of an innermost nest with 3-km grid spacing without a cumulus parameterization scheme produced the intensification that agreed best with observations. The medium-range forecast model (MRF) planetary boundary layer (PBL) scheme produces a PBL that is too dry and too deep as the winds increase toward hurricane strength. However, use of the Burk-Thompson PBL leads to excessive intensification with 9-km grid spacing. A noteworthy conclusion from the Davis and Bosart study is that the various choices of model physics produced storm intensities ranging from a marginal storm to a mature hurricane.

A recent simulation study of Hurricane Diana (Hendricks et al. 2004) examined the role of the so-called vortical hot towers in the formation of Diana. These vortical hot towers are cores of deep cumulonimbus convections possessing extreme heating rates (up to $200 \mathrm{~K} \mathrm{~h}^{-1}$ ) and vertical motions (up to $14 \mathrm{~m} \mathrm{~s}^{-1}$ ) that led to stretching of the column and spin-up of cyclonic vorticity right down to the surface. It is found that TC formation can be assisted by 
these hot towers through multiple mergers and axisymmetrization of the low-level potential vorticity anomalies associated with them.

As described in this brief literature review, only a few numerical studies on TC formation in the WNP exist. In particular, more studies of formations in the monsoon trough environment are needed, including at the eastern end of the trough (Kuo et al. 2001), since more than $75 \%$ of the global tropical cyclones form in the monsoon trough. Therefore, this study complements the previous ones by identifying suitable model configurations for TC formation simulations and predictions, and an understanding of the role of MCSs in TC formation. This study illustrates the effects of various model sensitivities on the formation of Typhoon Robyn (1993) when using a moderate-resolution model grid. There are two reasons for presenting results from moderate-resolution simulations. First, the $27-\mathrm{km}$ nest grid is comparable to the resolution of most of the current operational regional models, so that the model sensitivities found here should be applicable to those operational models. Second, this resolution is comparable to the separation of the dropwindsonde observations acquired during the formation of Typhoon Robyn in 1993 (more details in next section). Thus, when these observations are reanalyzed over the nested grid (see section $4 \mathrm{~b}$ ), the model simulations can be compared directly with these reanalyses. Higher-resolution simulations using the appropriate model configurations identified in this study, and more detailed diagnostic calculations associated with the simulation of Robyn formation, will be presented in a later report.

\section{FORMATION OF TYPHOON ROBYN}

Since detailed information about the life history of Typhoon Robyn can be found in ATCR (1993) and Harr et al. (1996a), only brief descriptions relevant to the subsequent discussions are given here. Typhoon Robyn (13W in ATCR) originated on 0000 UTC 31 July (hereafter 073100) from an area of enhanced convection near the equatorial trough at $7^{\circ} \mathrm{N}, 158^{\circ} \mathrm{E}$ (Fig. 1). A Tropical Cyclone Formation Alert (TCFA) was issued by the Joint Typhoon Warning Center (JTWC) at 073113 based on indications from satellite imagery and reconnaissance observations that a cyclonic circulation was developing. The first warning was then issued by JTWC at 080112 .

The synoptic situation before the formation of Robyn consisted of two low-level monsoon depressions at about $138^{\circ} \mathrm{E}$ and $155^{\circ} \mathrm{E}$, respectively (Fig. 2a). Strong cross-equatorial flow was south of both monsoon depressions. The cross-equatorial flows between $150^{\circ} \mathrm{E}$ and $160^{\circ} \mathrm{E}$ started to increase as early as 072400 , and reached a maximum between 072900 and 073100 just before the formation of Robyn (Harr et al. 1996a). Therefore, the enhanced convection in the eastern monsoon depression (letter $\mathrm{C}$ in Fig. 2a) is closely related to these strengthened low-level flows. Notice also that Robyn was forming near the eastern end of the monsoon trough where convergence between the trade easterlies and equatorial westerlies is known to be favorable for formation. At $200 \mathrm{hPa}$, a strong tropical upper-level tropospheric trough (TUTT) existed in the region between $150^{\circ} \mathrm{E}-160^{\circ} \mathrm{E}$ and $10^{\circ} \mathrm{N}-15^{\circ} \mathrm{N}$ (Fig. 2b). Divergent flow associated with this TUTT at $7^{\circ} \mathrm{N}, 158^{\circ} \mathrm{E}$ (letter D in Fig. 2b) was favorable for the formation of Robyn. Note that a similar divergence did not exist above the western monsoon depression. 


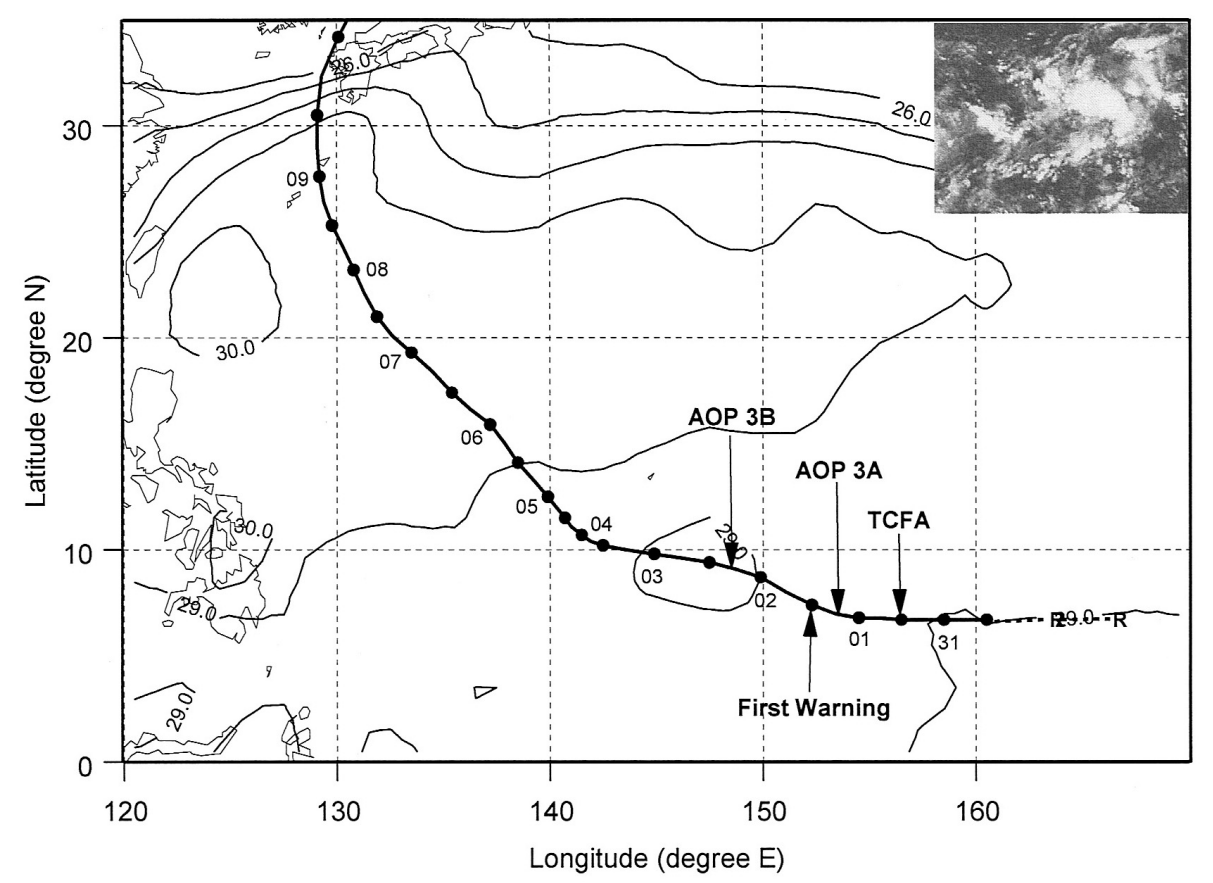

Fig. 1. Best track of Typhoon Robyn with the dates in July and August 1993. Background contour is the weekly mean sea-surface temperature $\left({ }^{\circ} \mathrm{C}\right)$ starting from 1 August 1993. Insert is a GMS-5 infrared satellite imagery at 1200 UTC 31 July 1993 (coverage about $140^{\circ}-160^{\circ} \mathrm{E}, 0^{\circ}-15^{\circ} \mathrm{N}$ ) showing the cloud clusters associated with the pre-Robyn disturbance.

Indeed, a relatively strong easterly flow was analyzed above that depression.

A mini-field experiment called Tropical Cyclone Motion (TCM-93) was carried out in the WNP to gather in situ measurements related to the effects of MCSs on TC tracks and formation (Harr et al. 1993). Several aircraft observing periods (AOPs) were carried out when Tropical Storm Ofelia (Harr et al. 1996b) and Typhoon Robyn (Harr et al. 1996a) formed. The two AOPs relevant to this study are AOP-3A and AOP-3B (see positions in Fig. 1). The 17 dropwindsondes deployed during AOP-3A centered around $153^{\circ} \mathrm{E}, 7^{\circ} \mathrm{N}$ help define the structure of the monsoon depression and of a MCS embedded in the large-scale circulation. During AOP-3B, two additional episodes of MCS development in the northern portion of the monsoon depression (around $149^{\circ} \mathrm{E}, 9^{\circ} \mathrm{N}$ ) were documented with 23 dropwindsondes.

Harr et al. (1996a) prepared gridded analyses with $1^{\circ}$ latitude/longitude resolution that incorporated the TCM-93 observations using a multiquadric interpolation technique with background fields from the Navy Operational Global Atmospheric Prediction System (NOGAPS) global analyses. The transformation of the eastern monsoon depression in Fig. 2a to a TC was defined by Harr et al. to occur in three stages. A large MCS first appeared along the eastern 
(a) NOGAPS 850-hPa flow at 12 UTC 31 Jul 1993

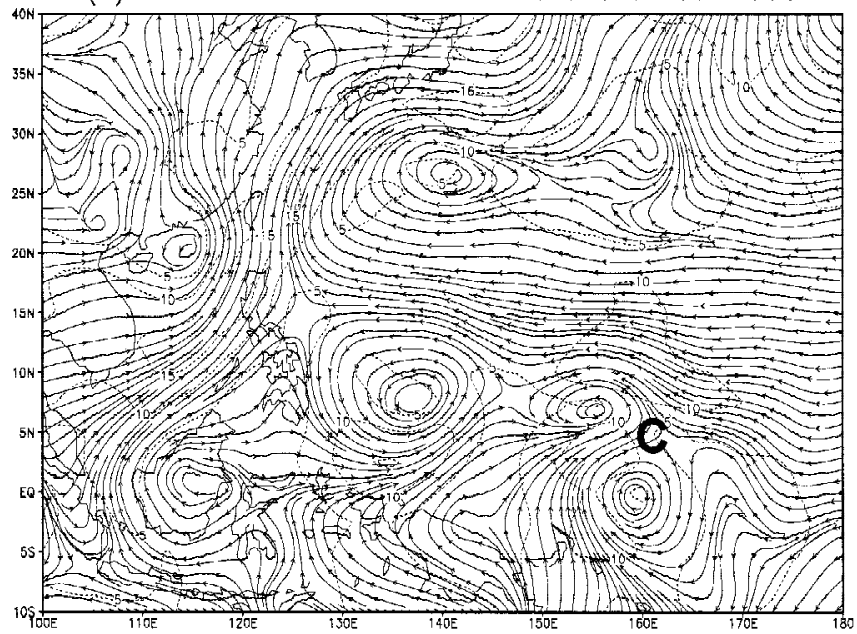

(b) NOGAPS 200-hPa flow ot 12 UTC 31 Jul 1993

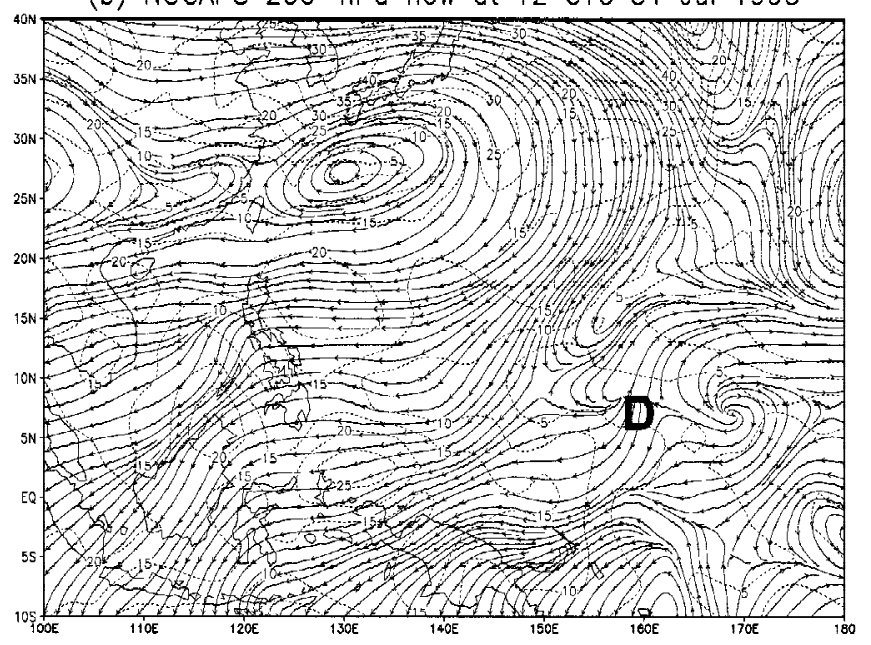

Fig. 2. (a) 850-hPa and (b) 200-hPa large-scale flow from NOGAPS analyses at 1200 UTC 31 July 1993 with wind speed (unit: $\mathrm{m} \mathrm{s}^{-1}$ ) in dashed contour. Letters $\mathrm{C}$ and $\mathrm{D}$ indicate the convergent and divergent locations, respectively.

periphery of the monsoon depression (labeled A in Fig. 3a) during the pre-organization stage described by the AOP-3A observations, which suggest that the MCS may have had a subsynoptic contribution to the vorticity structure of the total circulation via an axisymmetrization process. However, unsaturated downdrafts associated with the MCS also contributed to its rapid decay. 
(a) 0030 UTC

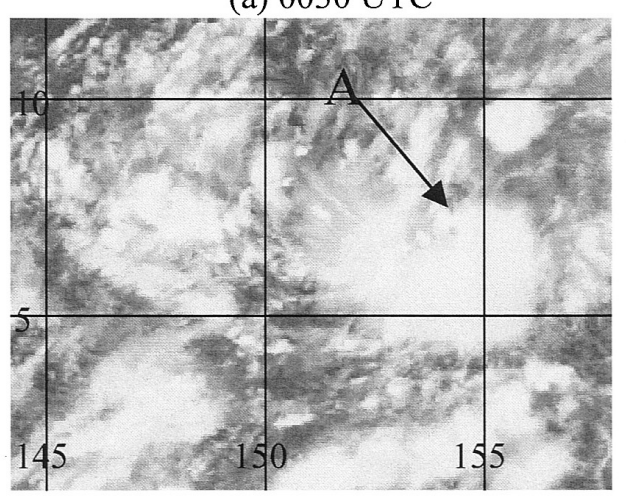

(b) 0630 UTC

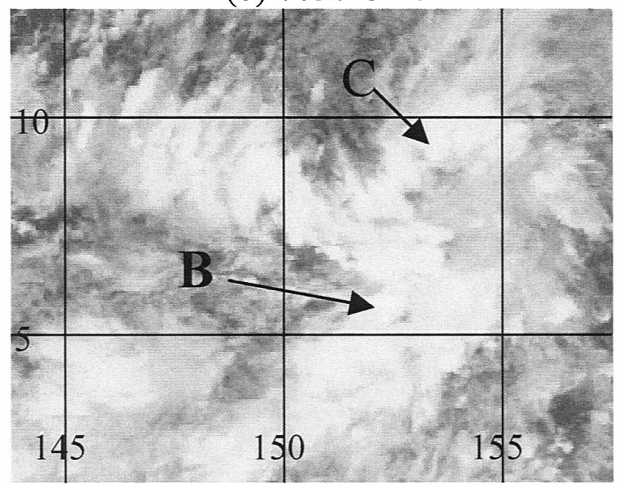

(c) 1030 UTC

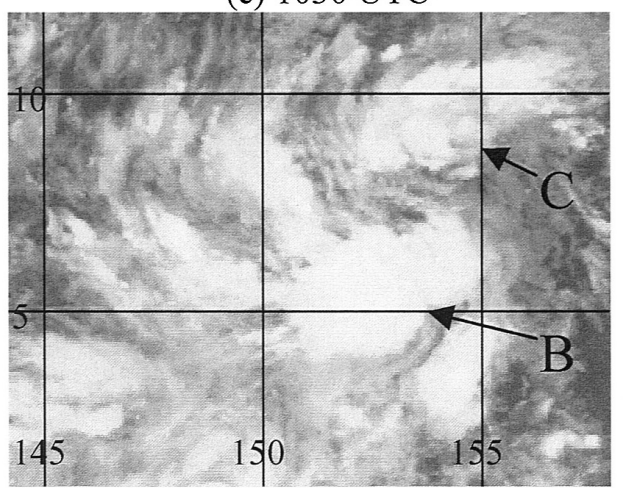

(d) 1330 UTC

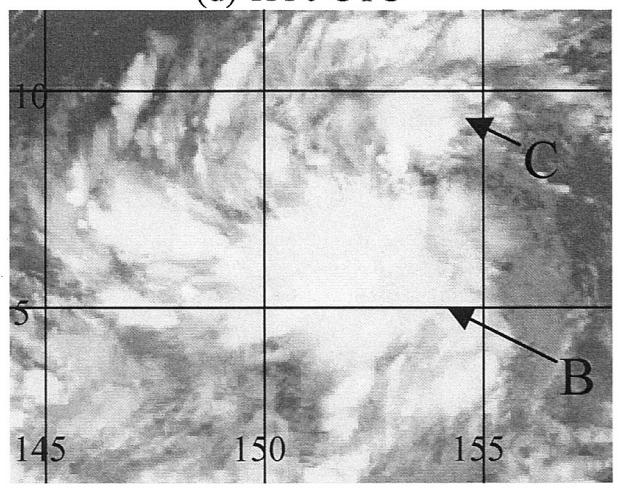

(e) 1730 UTC

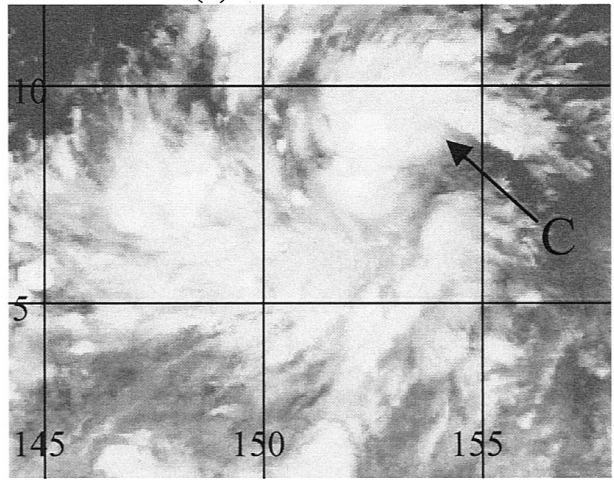

(f) 2130 UTC

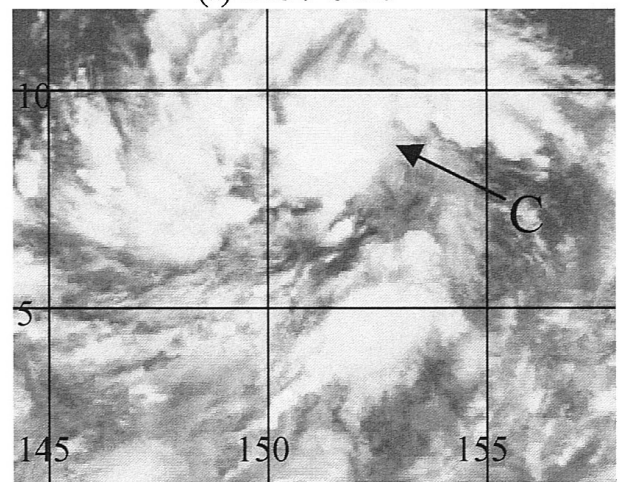

Fig. 3. GMS-5 infrared satellite imagery of the pre-Robyn disturbance at (a) 0030, (b) 0630, (c) 1030, (d) 1330, (e) 1730 and (f) 2130 UTC on 1 August 1993. Letters A, B, and C indicate three mesoscale convective system developments. 
During the $24 \mathrm{~h}$ between AOP-3A and AOP-3B, satellite imagery indicates that the interaction between the circulation and the convection began to increase. Although another MCS (labeled $\mathrm{B}$ in Fig. 3b) formed in a region of low-level convergence that was south of the previous MCS during AOP-3A, it also decayed rapidly. According to the analysis in Harr et al. (1996a), the monsoon depression circulation then seemed to intensify around a new MCS (labeled C in Fig. 3b) that had formed near the midlevel center of the monsoon depression. Eventually convection to the north and south began to organize into two broad principal bands (see Figs. 3c - f). Then the transformation to a tropical storm occurred during $\mathrm{AOP}-3 \mathrm{~B}$, which provided observations in a portion of the northern cloud band.

\section{MODEL CONFIGURATION AND EXPERIMENTS PERFORMED}

The PSU-NCAR MM5 model version 3 is used for the simulations. The model is started from 073112, which is right after the TCFA was issued. A 60-h integration, which includes the two AOPs during the formation of Robyn, is carried out until 080300. A coarse grid with 81$\mathrm{km}$ resolution and a nested grid with $27-\mathrm{km}$ resolution are used (Fig. 4). The coarse grid covers almost the entire WNP and includes the two monsoon depressions mentioned in section 2. The nested grid is centered on the pre-Robyn disturbance. Twenty-seven vertical levels with a model lid at $100 \mathrm{hPa}$ are used in the experiments. The initial conditions of the MM5 model are

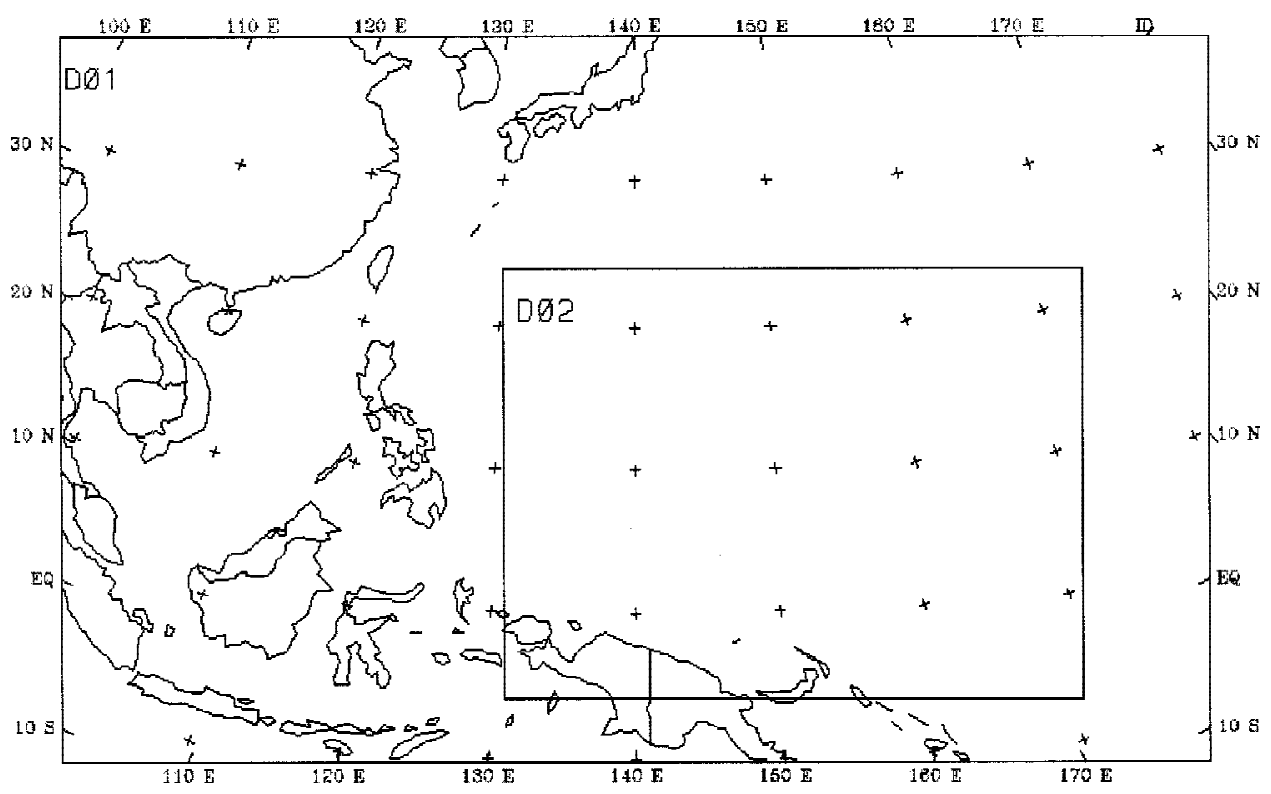

Fig. 4. The coarse $(81 \mathrm{~km})$ and nested $(27 \mathrm{~km})$ domains used in the MM5 simulations. 
obtained from NOGAPS analyses, and the SST in the control is the August 1993 monthly mean from the National Centers for Environmental Prediction (NCEP) reanalyses (Fig. 1). The Reynolds weekly mean SST with $1^{\circ}$ latitude resolution also produced by NCEP (Reynolds and Smith 1994) is also tested. Because the weekly SSTs produced more or less the same degree of intensification of Robyn during the integration, the monthly mean SST is retained throughout the experiments.

Since one of the foci of this study is model sensitivity in simulating a TC formation, a series of experiments (Table 1) is carried out utilizing the different physics options in MM5. Much of the attention is on the sensitivity to the cumulus parameterization, PBL parameterization, and the explicit moisture scheme. Then some of the experiments are com-

Table 1. Configurations of the experiments performed in this study.

\begin{tabular}{|c|c|c|c|c|}
\hline $\begin{array}{c}\text { Experiment } \\
\text { Name }\end{array}$ & $\begin{array}{c}\text { Cumulus } \\
\text { Parameterization }\end{array}$ & PBL Scheme & $\begin{array}{l}\text { Explicit } \\
\text { Moisture }\end{array}$ & $\begin{array}{c}\text { Moist } \\
\text { Diffusion }\end{array}$ \\
\hline $\begin{array}{c}\text { BM1 } \\
\text { (control) }\end{array}$ & Betts-Miller $^{1}$ & MRF & Reisner & Yes \\
\hline \multicolumn{5}{|c|}{ Sensitivity to cumulus parameterization } \\
\hline $\mathrm{BM} 2$ & Betts-Miller ${ }^{2}$ & MRF & Reisner & Yes \\
\hline GRL & Grell & MRF & Reisner & Yes \\
\hline KF & Kain-Fritsch & MRF & Reisner & Yes \\
\hline \multicolumn{5}{|c|}{ Sensitivity to PBL parameterization } \\
\hline BTS & Betts-Miller & Burk-Thompson & Reisner & $\mathrm{No}^{3}$ \\
\hline ETA & Betts-Miller & eta & Reisner & $\mathrm{No}^{3}$ \\
\hline GSM & Betts-Miller & Gayno-Seaman & Reisner & $\mathrm{No}^{3}$ \\
\hline \multicolumn{5}{|c|}{ Sensitivity to explicit moisture scheme } \\
\hline SIMICE & Betts-Miller & MRF & Simple Ice & Yes \\
\hline MrX & Betts-Miller & MRF & Mixed-Phase & Yes \\
\hline Schultz & Betts-Miller & MRF & Schultz & Yes \\
\hline
\end{tabular}

${ }^{1}$ Standard reference profile

${ }^{2}$ Tropical reference profile

${ }^{3}$ No moist diffusion available other than the MRF PBL scheme 
pared with the observations taken during the TCM-93 AOPs.

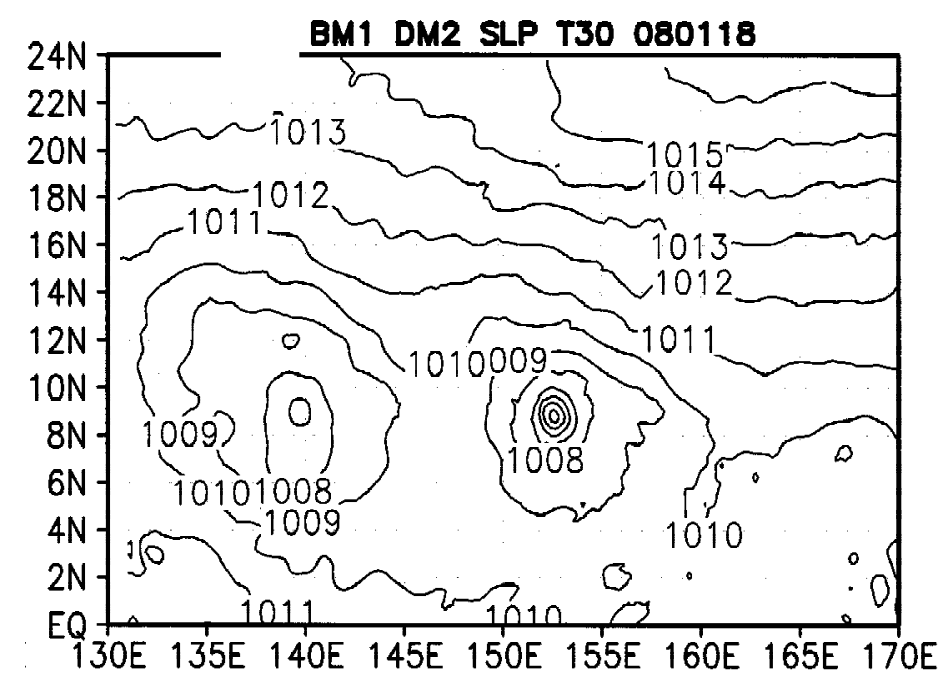

Fig. 5. Simulated mean-sea-level pressure in the control experiment (BM1) after $30 \mathrm{~h}$ of simulation to show the early intensification stage of Typhoon Robyn.

\section{THE CONTROL SIMULATION}

\subsection{Physical Processes Identified in the Control Simulation}

In the control experiment (BM1), the vortex intensification is greater than in other experiments, and is closest to that observed for Typhoon Robyn. Therefore, some brief descriptions of the BM1 results are first given based on some three-hourly outputs from the model. The BM1 experiment utilizes the Betts-Miller cumulus parameterization with a standard "midlatitude" profile with pressure deficit values of $-38.75,-58.75$ and $-18.75 \mathrm{hPa}$, the PBL parameterization as in the NCEP MRF model, and the Reisner explicit moisture scheme with moisture diffusion. Three hours after the start of the simulation (073115), the sea-level pressure (SLP) consisted of a trough at about $3-6^{\circ} \mathrm{N}, 145^{\circ} \mathrm{E}$. As mentioned in section 2, two monsoon depressions began to develop at about $138^{\circ} \mathrm{E}$ and $155^{\circ} \mathrm{E}$. After about $18 \mathrm{~h}$ of simulation, the eastern monsoon depression deepened further, and later developed into a tropical depression (Fig. 6). Throughout the 60-h simulation period, the minimum SLP of the simulated cyclone agrees well with that reported in the best track of Robyn (see the corresponding line in Fig. 15a), which decreases to about $1005 \mathrm{hPa}$ after $30 \mathrm{~h}$ and $990 \mathrm{hPa}$ at the end of the period.

As discussed in Harr et al. (1996a), the intensification of the monsoon depression near $155^{\circ} \mathrm{E}$ was closely related to the low-level cross-equatorial wind flow near that longitude. This 


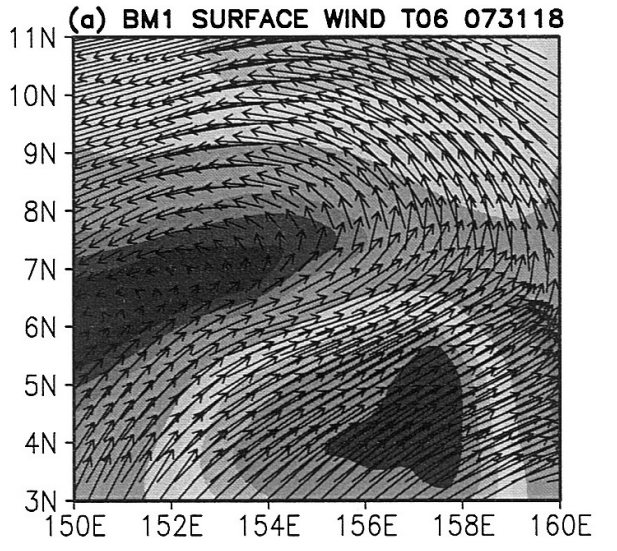

10

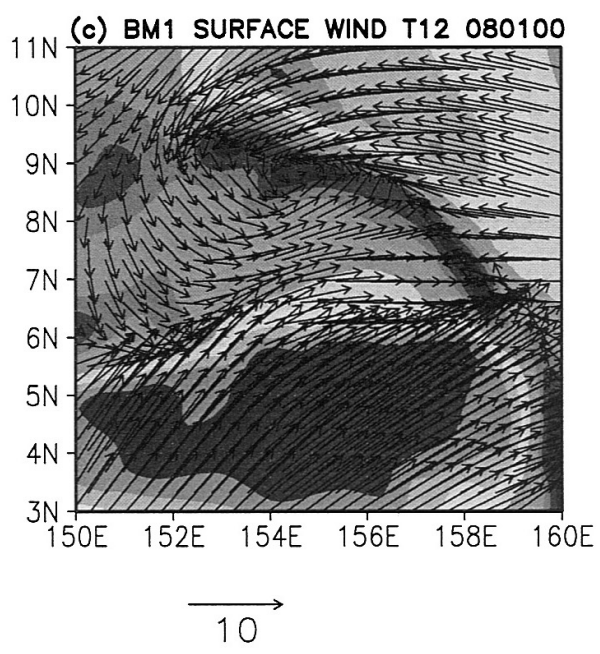

(b) BM1 SURFACE WIND TO9 073121

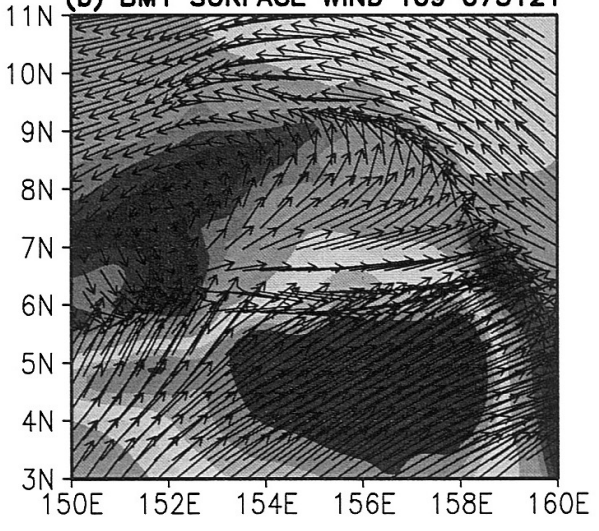

10

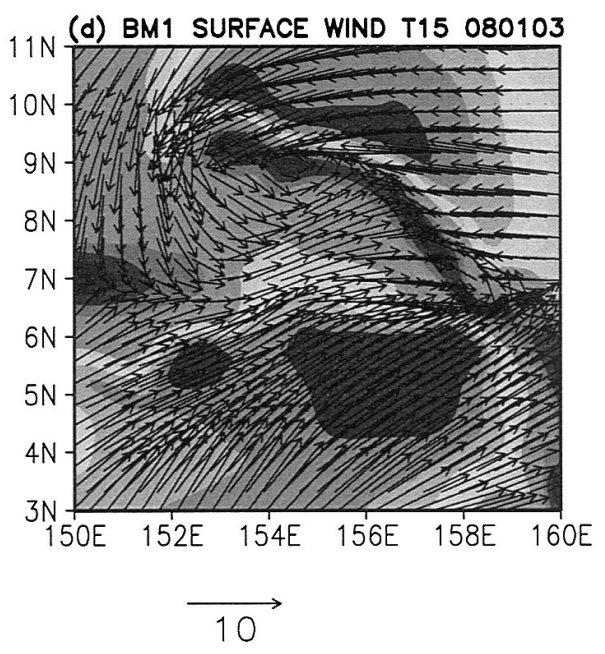

Fig. 6. Simulated wind at lowest model level in the control experiment (BM1) at (a) $6 \mathrm{~h}$, (b) $9 \mathrm{~h}$, (c) $12 \mathrm{~h}$ and (d) $15 \mathrm{~h}$, and shaded contours indicate magnitudes. The reference arrow is $10 \mathrm{~m} \mathrm{~s}^{-1}$, contour interval is $1 \mathrm{~m} \mathrm{~s}^{-1}$, and the dark shaded area over the lower latitudes indicates southwesterlies with magnitudes larger than $11 \mathrm{~m} \mathrm{~s}^{-1}$.

cross-equatorial flow is also in the MM5 simulations (Fig. 6). At 073118, the wind maximum at the lowest model level is at $4^{\circ} \mathrm{N}-6^{\circ} \mathrm{N}, 156^{\circ} \mathrm{E}-158^{\circ} \mathrm{E}$ (Fig. 6a). This area of strong southwesterlies increases further and persists for the next $9 \mathrm{~h}$ (Figs. $6 \mathrm{~b}-\mathrm{d}$ ). Due to these increased surface southwesterlies, a convergence line is simulated at about $6^{\circ} \mathrm{N}$. The line is formed in association with the cyclonic winds of the monsoon depression to the north, which also increases in magnitude and forms another convergence line at about $9^{\circ} \mathrm{N}$. 
An east-west oriented line of convection is then triggered by the surface convergence. The three-hourly accumulated convective rain starts to increase after 073118 at the eastern end of the southwesterly surge area (Fig. 7a), which indicates that it is the first convective center in the development of Typhoon Robyn. In the subsequent nine hours (Figs. $7 \mathrm{~b}-\mathrm{d}$ ), the convective rain areas extend to the southern and northern convergence lines mentioned above. Vertical velocities at $300 \mathrm{hPa}$ (Fig. 8) are consistent with the convective rain field. Deep convection occurs first at the intersection of the two low-level convergence lines at about 080100 (Fig. 8a), and persists for the next three hours (Fig. 8b). At 080106 (Fig. 8c), two new centers form at about $9^{\circ}-10^{\circ} \mathrm{N}, 152^{\circ} \mathrm{E}$, and $6^{\circ} \mathrm{N}, 153^{\circ} \mathrm{E}$. The vertical velocity associated with the center to the north increases further in the next three hours (Fig. 8d). In addition, convection also occurs in a broad band in the rain field consistent with the northern convergent line, which is probably due to the advection of the easterlies in the monsoon depression.
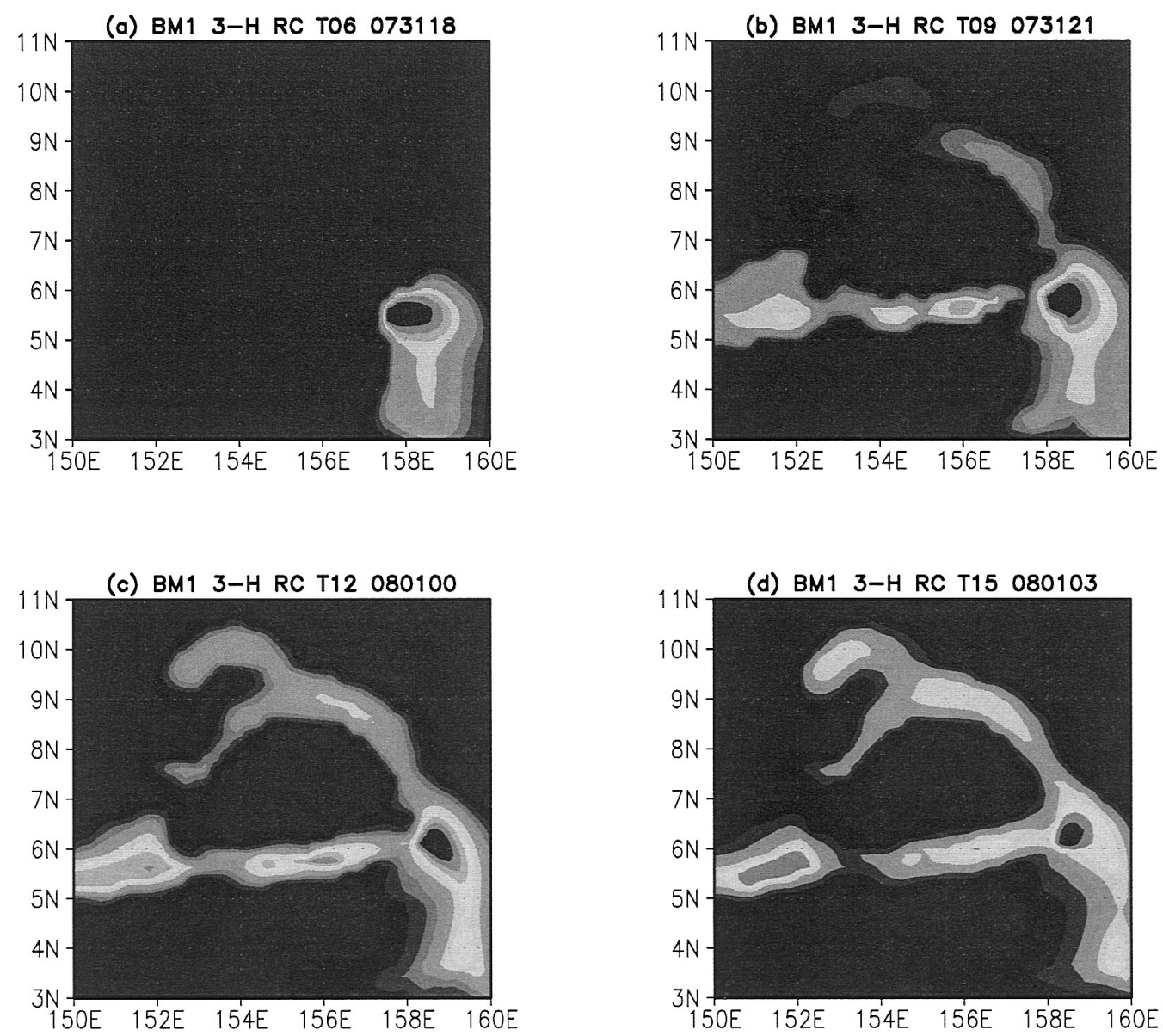

Fig. 7. As in Fig. 6, except for three-hourly accumulated convective rainfall. The contour interval is $1 \mathrm{~cm}$. 
(a) BM1 300-hPa WEL T12 080100

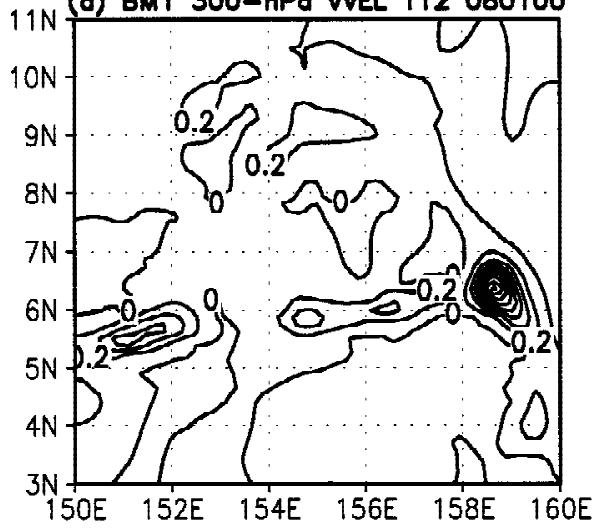

(c) BM1 300-hPa WEL T18 080106

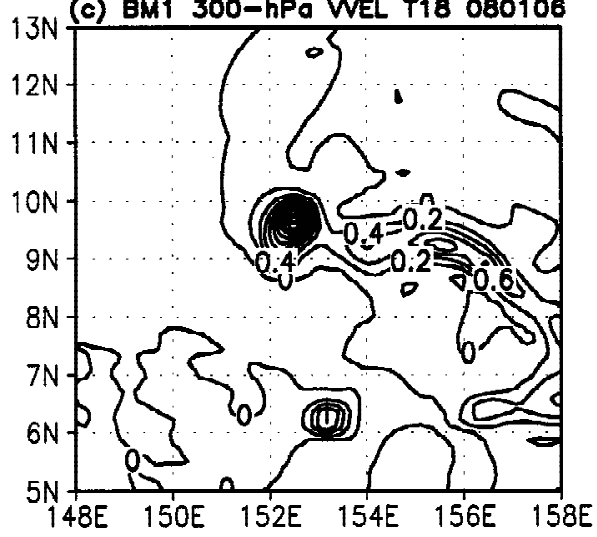

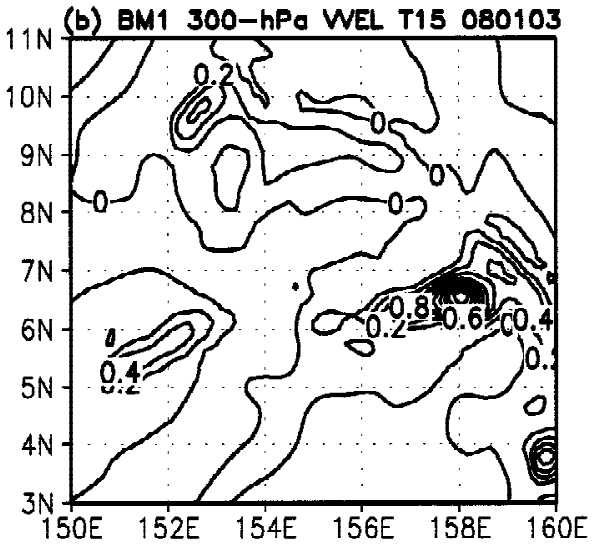

(d) BM1 300-hPo WEL T21 080109

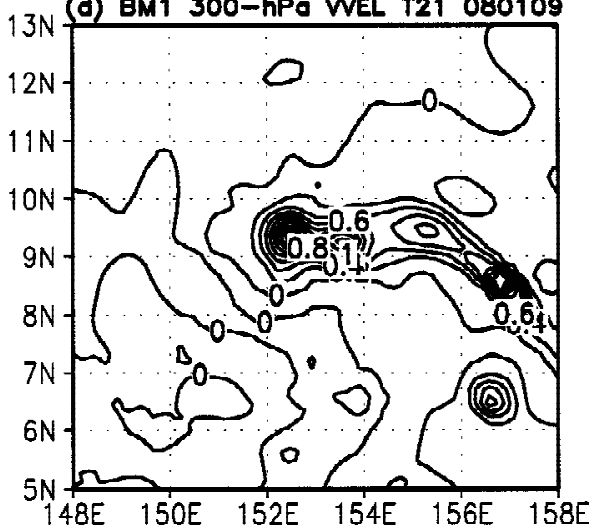

Fig. 8. As in Fig. 6, except for 300-hPa vertical velocity (unit: $\mathrm{m} \mathrm{s}^{-1}$ ).

Updrafts associated with rainfall in deep convection are a possible mechanism for cyclonic relative vorticity generation (Chen and Frank 1993). At 080100 in the MM5 simulation (Fig. 9a), the 850-hPa relative vorticity field has high values along the two low-level convergence lines, and in particular at the convection center at about $159^{\circ} \mathrm{E}$. A vertical cross section (meridional plane) of the potential vorticity (PV) at this longitude reveals a mid-level PV maximum (Fig. 9a, right panel) at around $400 \mathrm{hPa}$. The $850-\mathrm{hPa}$ vorticity pattern and the PV vertical structure at almost the same longitude are similar in the next three hours (not shown). At 080106 (Fig. 9b), the low-level vorticities associated with the convection centers previously identified in the rain field and vertical velocity further increase. The PV column at $152.5^{\circ} \mathrm{E}$ has already deepened to near the surface (right panel of Fig. 9b). Note also the PV maximum between $6^{\circ} \mathrm{N}$ and $7^{\circ} \mathrm{N}$ that is associated with the southern convection center. Near the eastern end of the vorticity band, a PV center at about $158.5^{\circ} \mathrm{E}$ is also simulated that has similar PV 

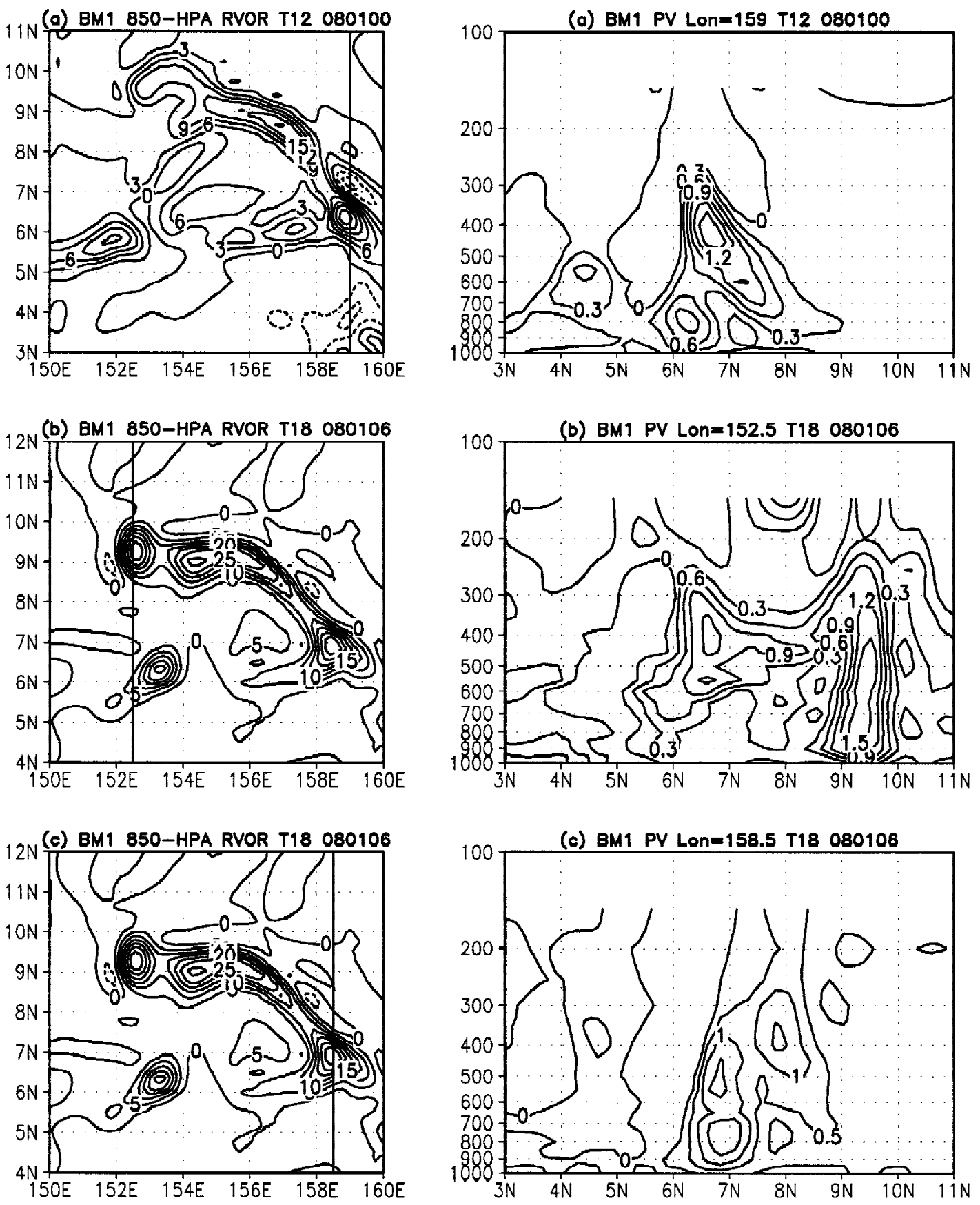

Fig. 9. Simulated 850-hPa relative vorticity (left panels) and corresponding vertical cross sections of potential vorticity along the indicated longitudes (right panels) at five times. Units are $10^{-5} \mathrm{~s}^{-1}$ and PVU, respectively. 

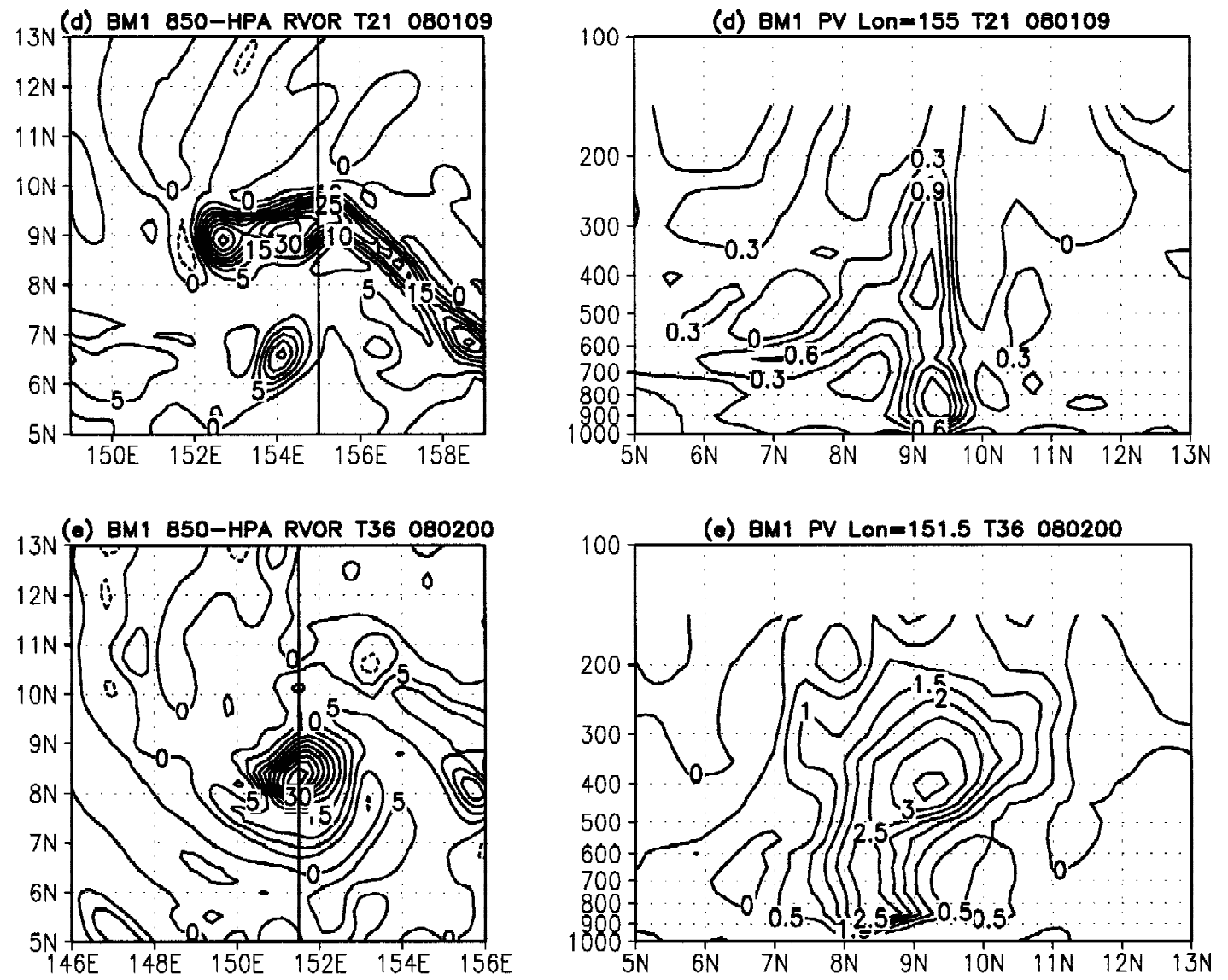

Fig. 9. Continued.

values as in the western one (Fig. 9c). Three hours later (Fig. 9d), the system starts to 'axisymmetrize' into a single vorticity center. The southern center is still isolated, and this feature dissipates subsequently. Notice the PV column at the center of the system has already developed to near the surface (Fig. 9d, right panel). The entire system is also advected by the easterlies, such that at 080200 (Fig. 9e) the low-level vorticity center is at about $151^{\circ} \mathrm{E}$, and the vertical PV structure has strengthened and expanded in horizontal scale (Fig. 9e, right panel).

Mid-to-upper-level warm anomalies are also simulated by the model in association with the convective events identified. A slight warm anomaly already appears at 080100 within the first convection center (Fig. 10a). Warm anomalies later develop in the western convection (Figs. 10b). A slightly warm region is also found at about $6.5^{\circ}$ latitude that may be associated with MCS B. As mentioned in section 2, this convection does not develop further. When a mature warm-core center is simulated at 080200 (Fig. 10c), the potential temperature anomaly at the center of the system is nearly $2 \mathrm{~K}$. 


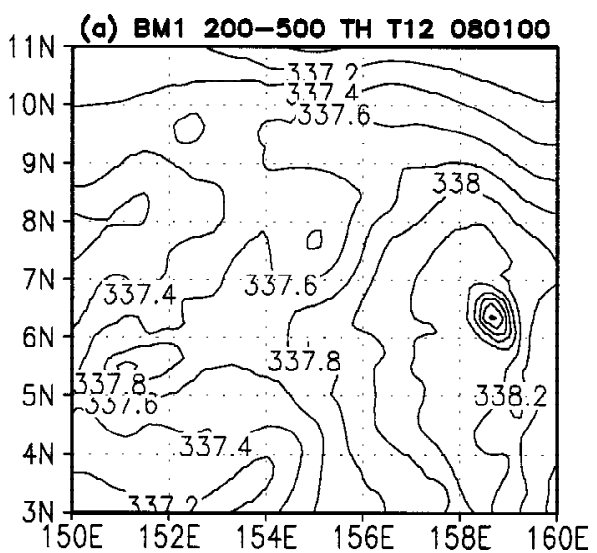

(b) BM1 200-500 TH T18 080106

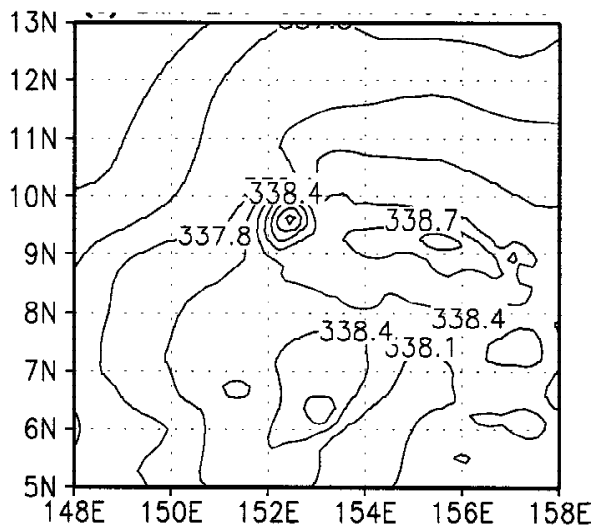

(c) BM1 200-500 TH T36 080200

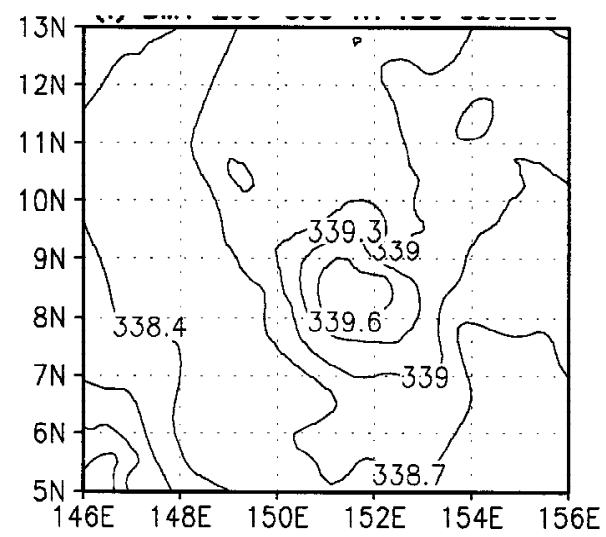

Fig. 10. As in Fig. 6, except for 200-500-hPa averaged potential temperature at (a) $12 \mathrm{~h}$, (b) $18 \mathrm{~h}$ and (c) $36 \mathrm{~h}$ of simulation. 


\subsection{Comparisons with the TCM-93 Observations}

Since the control experiment best simulates the intensification observed in Typhoon Robyn, further comparisons are made with the observations during the AOP-3A that was carried out just before Robyn intensified to a tropical depression. First, the control simulation roughly agrees with the cloud system development as depicted in the satellite imagery (Fig. 3). The first convection center appears at about 080100 in response to the strengthened southwesterlies, and the low-level vorticity maxima in Figs. 9 and 10 generally correspond to the MCS A, although the simulated location is about $3^{\circ}$ longitude too far east. At 080106 , a second convection center develops at about $6^{\circ} \mathrm{N}$, which also possesses a mid-level PV maximum (Fig. 9b). This center might be considered to correspond to MCS B because this convection was also only sustained for a short time and did not develop significantly. The MCS C, which eventually develops into Robyn, corresponds to the convective center between $9^{\circ} \mathrm{N}$ and $10^{\circ} \mathrm{N}$ that appears in the simulation at around 080106 . This center is sustained and eventually intensifies into a tropical depression. Thus, the control simulation is able to reproduce quite well both spatially and temporally the successive convective events during the formation of Robyn, which may be surprising given the relatively coarse resolution and with only large-scale analyses as inputs.

Harr et al. (1996a) studied the formation of Typhoon Robyn by constructing reanalyses using a multiquadric interpolation technique to modify an initial guess from NOGAPS analyses with the aircraft observations from AOP-3A and AOP-3B, and some soundings released from the islands in the vicinity. A similar procedure is followed in this study by using the objective analysis component in MM5 with the same multiquadric interpolation technique, and enforcing full acceptance of the AOP-3A observations (i.e., no further quality control is used). Since the AOP-3A was centered at about 080104 (with a time span of about six hours), the adjacent NOGAPS analyses are interpolated to this time to be used as the background field.

One anomalous feature in the control simulation is that the cyclonic circulations at 850 and $500 \mathrm{hPa}$ are not aligned vertically as in the Harr et al. (1996a) analyses. The 16-h forecast 500-hPa wind fields valid at 080104 do have a similar horizontal structure as in the analysis with a center at about $6.5^{\circ} \mathrm{N}, 152^{\circ} \mathrm{E}$ (Fig. $11 \mathrm{~b}$ ). However, the 850 -hPa streamlines are distorted by mesoscale circulations that have been generated by the simulated convective events. The cyclonic flow near $7^{\circ} \mathrm{N}, 158^{\circ} \mathrm{E}$ is considered to be related to MCS A, while the convergent flow near $6^{\circ} \mathrm{N}, 153^{\circ} \mathrm{E}$ is close to where MCS B originates. The overall monsoon depression circulation is shifted a little to the north in the simulation, which corresponds more to the position where MCS C is developing. This implied vertical tilt of the simulated monsoon depression is maintained until about 080112, when the circulation associated with MCS C becomes stronger such that the cyclonic column becomes centered on it.

A particular feature of the MCSs identified in Harr et al. (1996a) was that a low-level dry region often accompanied the MCS developments. These dry regions usually formed under the stratiform rain region of MCSs where unsaturated downdrafts occurred. This kind of moisture profile is identified in dropsondes 8 and 15 from AOP-3A, which are near the center of the monsoon depression. A similar dry region is simulated in the MM5 model on the western side 
(a) BM1 DM2 850-hPo Flow T16 93080104

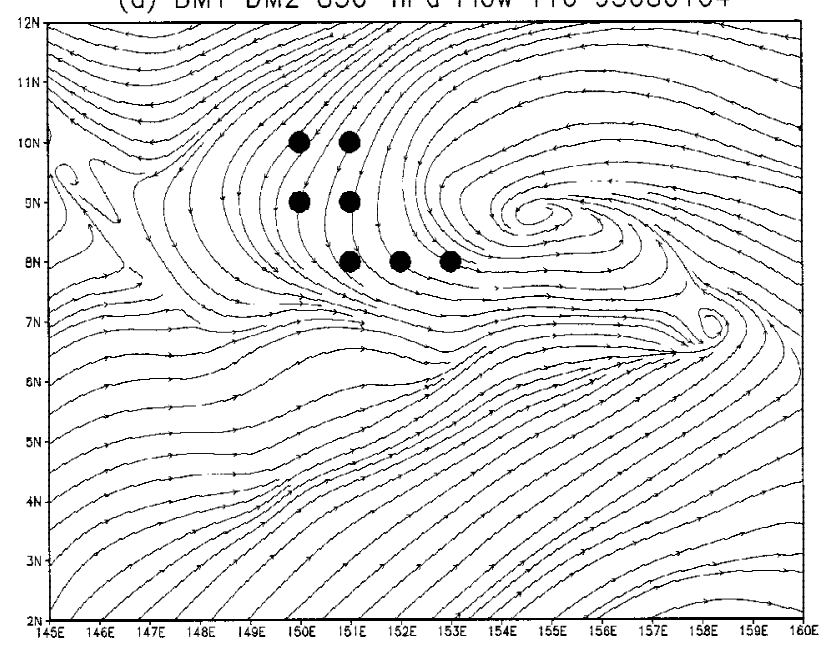

(b) BM1 DM2 500-hPa Flow T16 93080104

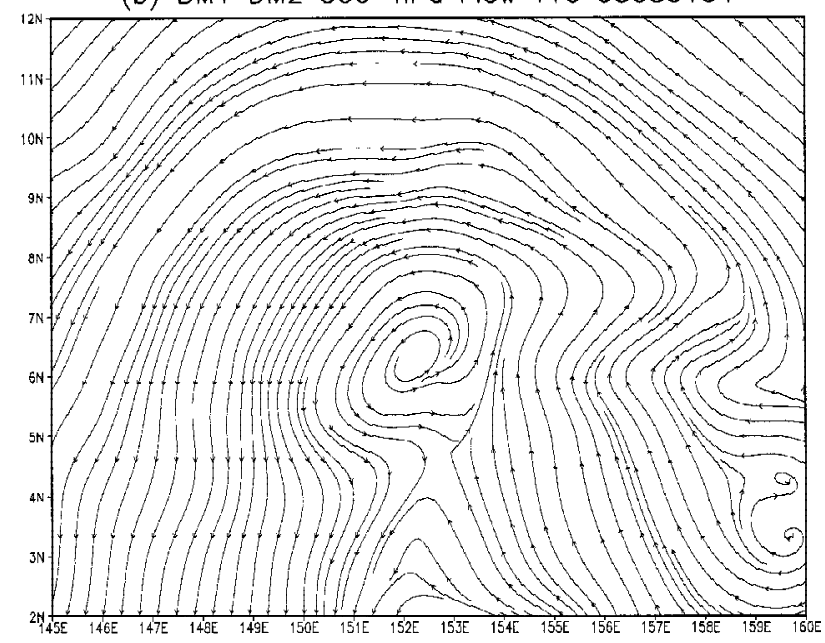

Fig. 11. Simulated 850-hPa and 500-hPa flows at 080104 in experiment BM1. The dots indicate the locations where a similar vertical thermodynamic profile as in Fig. 12 is found.

of the monsoon depression (dots in Fig. 11a). A simulated sounding near $8^{\circ} \mathrm{N}, 151^{\circ} \mathrm{E}$ (Fig. 12) has a dry layer between 900 and $700 \mathrm{hPa}$ underneath a moist layer. This contrast in the location of this dry region may be because dropsondes 8 and 15 are closer to MCS A so that the dry region is generated by this first MCS development. As mentioned, the simulated MCS A in the model is east of the observed position, and is quickly replaced by the MCS C development. Thus, the identified dry region in the simulation is likely related to the MCS C that starts to 
develop earlier than observed from satellite imagery.

Harr et al. (1996a) note that the monsoon depression in which Robyn developed was associated with upper-level updrafts and low-level downdrafts (Fig. 13a). As can be seen in Fig. 7 of Harr et al. (1996a), the center of the monsoon depression is between $152^{\circ} \mathrm{E}$ and $153^{\circ} \mathrm{E}$. Thus, the updrafts are at the periphery of the depression while the downdrafts are mainly at the center in the analysis. The consequence is an overall stretching effect that is beneficial for cyclonic vorticity generation. In the MM5 model, no explicit downdraft is simulated underneath the updrafts, but reduced vertical velocities are found between $155^{\circ} \mathrm{E}$ and $156^{\circ} \mathrm{E}$. However, note that the cross section in Fig. $13 \mathrm{~b}$ is at the low-level center $\left(8.5^{\circ} \mathrm{N}\right)$ in the simulation. As the simulated low-level processes are different from those depicted in the Harr et al. analysis, the vertical velocity field is also expected to deviate quite substantially from the corresponding analysis.

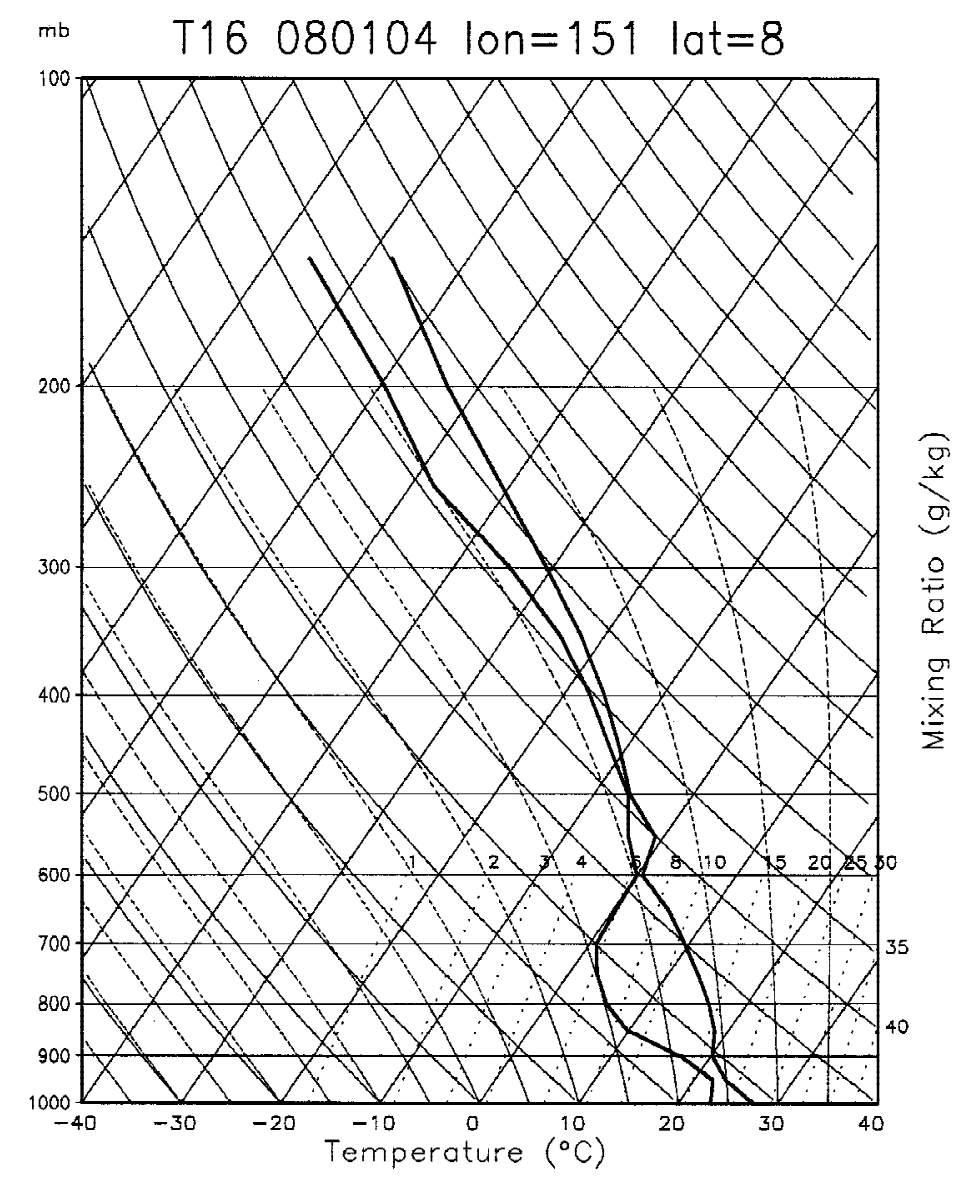

Fig. 12. Skew T-log p plot of temperature and dew point at $8^{\circ} \mathrm{N}, 151^{\circ} \mathrm{E}$ from experiment BM1 at 080104. 
(a) Analyzed theta_e and U-W winds 080104 lat $=6.5$

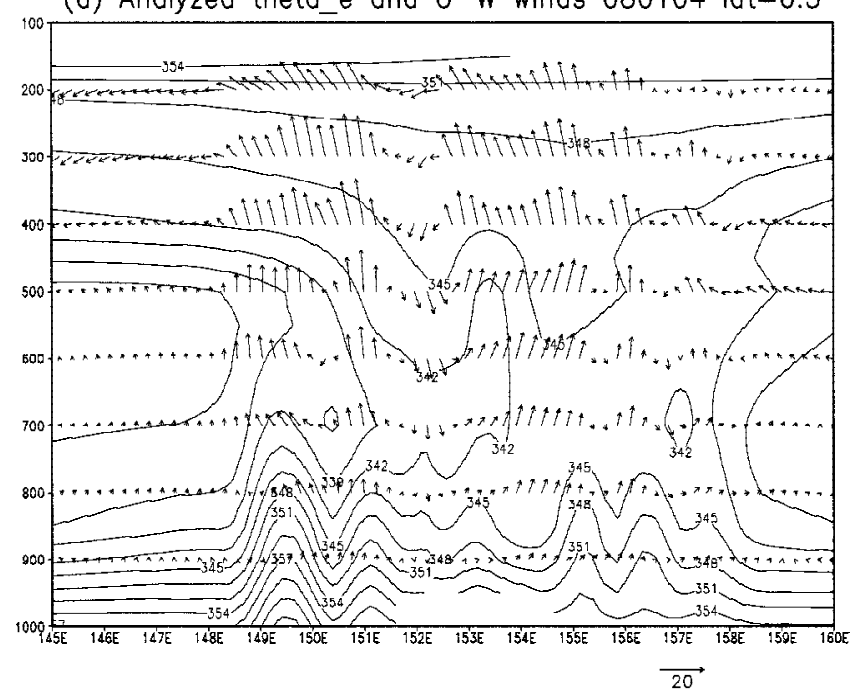

(b) BM1 theta_e and U-W winds T16 080104 lat $=8.5$

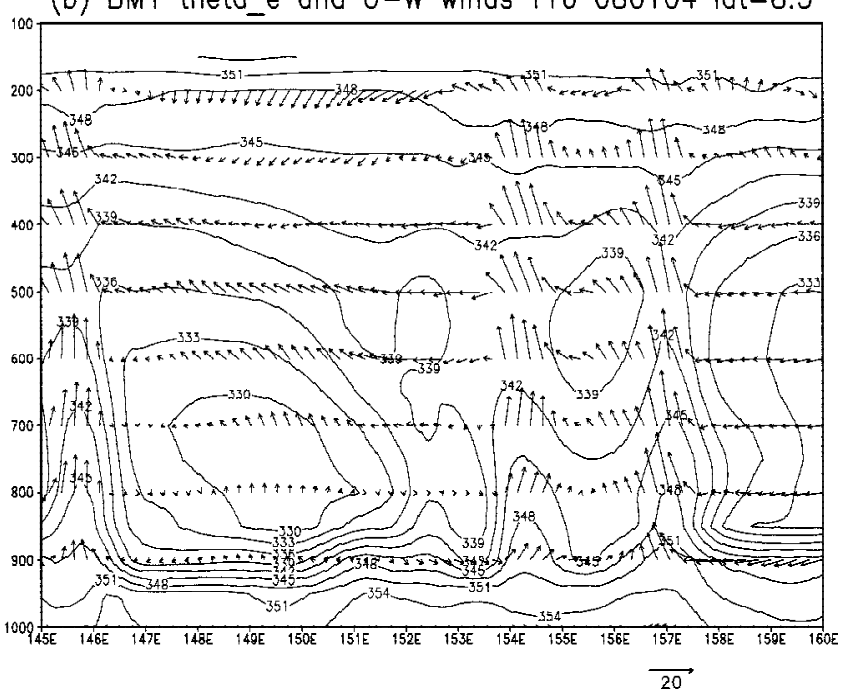

Fig. 13. Vertical cross section of equivalent potential temperature (unit: K) and zonal (unit: $\mathrm{m} \mathrm{s}^{-1}, 20 \mathrm{~m} \mathrm{~s}^{-1}$ reference arrow shown) and vertical winds (unit: $\mathrm{cm} \mathrm{s}^{-1}$, same reference arrow in $\mathrm{cm} \mathrm{s}^{-1}$ ) at (a) $6.5^{\circ} \mathrm{N}$ from analyzed flow with AOP-3A observations and (b) $8.5^{\circ} \mathrm{N}$ from experiment BM1 at 080104.

\subsection{Further Comments on the MCS Developments}

From the descriptions of the evolution of MCSs A, B, and C, it can be seen that their system development fits loosely into the Zehr (1992) two-stage development conceptual model. 
The first stage consists of the generation of MCSs A and B with only weak mesovortices, and then MCS C developed in the second stage when further deep convection occurred and MCS $\mathrm{C}$ developed near the center of the monsoon depression. A key feature of Zehr's conceptual model is a surge from the equatorial westerlies that triggers the second deep convection. A hypothesis here is that the "surge" is not necessarily from the large-scale flow, but is provided by the mutually interacting MCSs. In this case of Robyn's formation, there was not a second southwesterly surge other than that which occurred between 072900 and 073100 . In addition, MCS C developed at the northern periphery of the monsoon depression where it should not be triggered by southwesterlies.

Examination of the vertical wind shear environment during the formation of Robyn can also reveal more clearly the evolution of the three associated MCSs. At 080104, the 200-850$\mathrm{hPa}$ vertical shear appears as a divergent flow over MCS A near $158^{\circ} \mathrm{E}, 6.5^{\circ} \mathrm{N}$ (Fig. 14). However, MCS B at about $153^{\circ} \mathrm{E}, 6^{\circ} \mathrm{N}$ has strong vertical shear of nearly $20 \mathrm{~m} \mathrm{~s}^{-1}$, which is not favorable for its further development. In contrast, MCS C that developed near $154^{\circ} \mathrm{E}, 9^{\circ} \mathrm{N}$ was under weak-to-moderate easterly shear (which is favorable for MCV generation). The

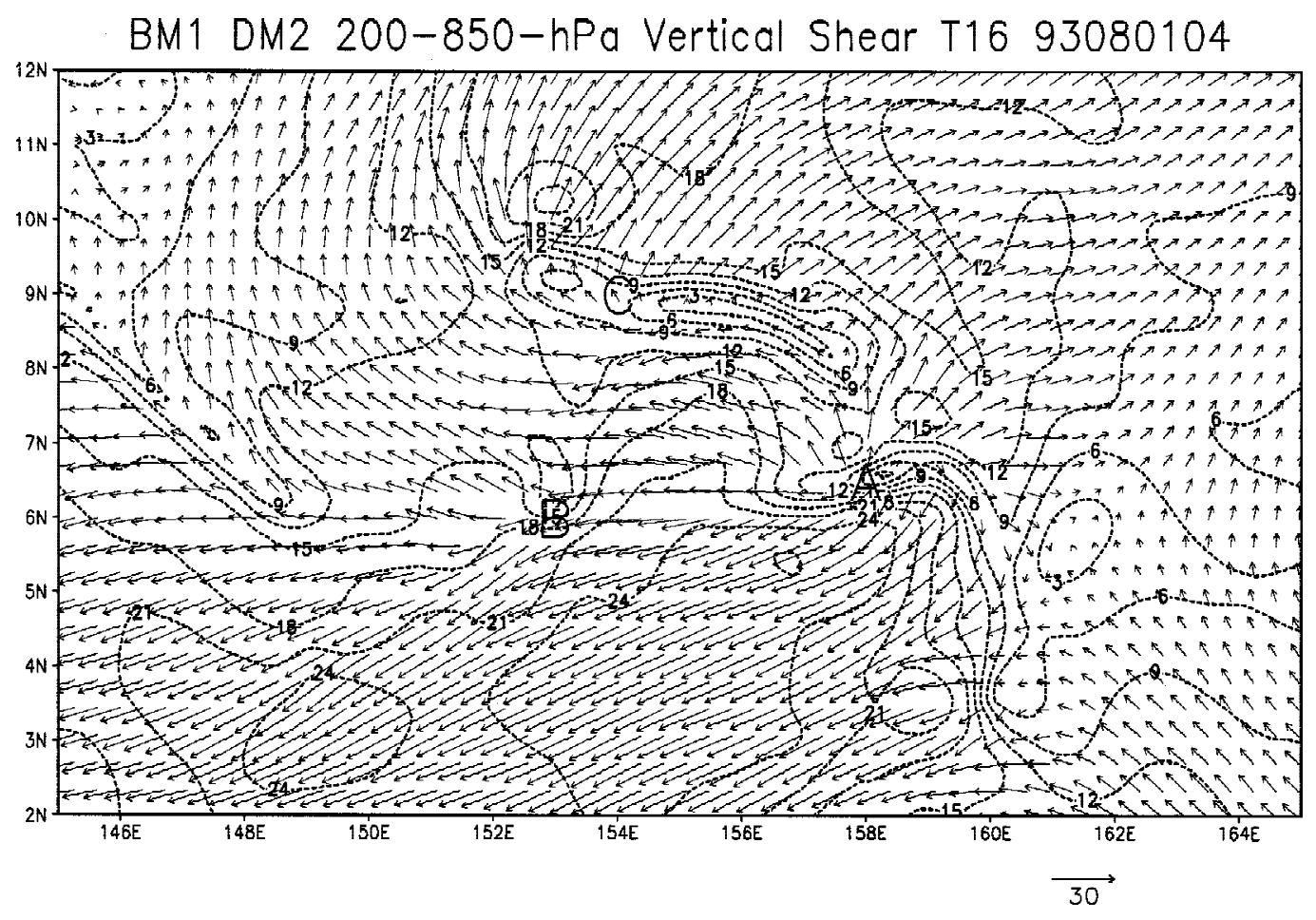

Fig. 14. Simulated 200-850-hPa vertical wind shear $\left(\mathrm{m} \mathrm{s}^{-1}\right)$ at 080104 with contour interval of $3 \mathrm{~m} \mathrm{~s}^{-1}$. Letters A, B and C denote the approximate positions of the three MCSs that developed during the formation of Typhoon Robyn. 
direction of the shear is also consistent with the location of the associated MCV (vorticity maximum west of the MCS, see Fig. 9b) and that of the lower-tropospheric dry region found in Fig. 11a. Thus, the conclusion is the favorable environment for the development of MCS C into a tropical depression is provided at least partially by the outflow structure from MCS A.

\section{MODEL SENSITIVITIES}

\subsection{Sensitivity to Cumulus Parameterization}

Three other experiments are performed in this study using different cumulus parameterizations. The Betts-Miller parameterization is basically an adjustment scheme that tends to adjust the saturation pressure deficits toward a reference profile specified at three levels: near the cloud base, the freezing level, and near the tropopause (Davis and Bosart 2002). In the standard Betts-Miller parameterization in the MM5 (as used in the control simulation BM1), the reference pressure deficits are $-38.75,-58.75$, and $-18.75 \mathrm{hPa}$ respectively at the three levels, which are representative of midlatitudes. Another Betts-Miller experiment (BM2) is performed that uses a 'tropical' profile with the pressure deficit values of $-20,-40$, and $-20 \mathrm{hPa}$ (Table 1). In addition, simulations are also carried out with the Kain-Fritsch scheme and the Grell scheme (named KF and GRL in Table 1). The Kain-Fritsch scheme involves explicit computations of a cloud model, and implements entrainment and detrainment between the cloud and the environment as a function of the buoyancy characteristics of various mixtures of clear and cloudy air. The Grell scheme utilizes a single cloud with updraft and downdraft fluxes and compensating motion to determine the heating and moistening profile.

The choice of cumulus parameterization largely determines the degree of intensification of Typhoon Robyn. Among the experiments, BM1 has the closest deepening of the central minimum pressure to that of the best track (Fig. 15a). Experiment GRL has a similar intensification profile as in BM1, but underestimates Robyn's intensity throughout the simulation. Both of the BM2 and KF simulations almost stop intensifying the TC after $42 \mathrm{~h}$. These differences in the simulated intensity from these experiments are not due to different SST values along the simulated tracks. After some erratic early tracks due to the appearance of a new convection center at the northern side, most of the tracks are not that different (Fig. 15b). The exception is the KF simulation in which the track takes a too northward orientation. Besides $\mathrm{KF}$, the other simulated tracks can basically reproduce the observed motion of Robyn up to 080300, although with a slight slow bias while Robyn was being steered steadily to the west.

The different degree of intensification when using different cumulus parameterization schemes is correlated with the simulated precipitation amounts. Experiments BM1 and KF have much larger three-hourly total convective rainfalls summed over the inner domain than those of BM2 and GRL. When the convective rainfall amount is restricted to within an area of approximately $780 \times 1050 \mathrm{~km}$ centered on Robyn, the differences among the schemes are reduced, but the BM1 experiment still has the largest amount, and BM2 has the least (Fig. 16).

An apparent inconsistency exists in that the KF scheme produces a similar convective rainfall amount as in BM1, but smallest intensification is simulated in the KF experiment. However, the convective rainfall in KF is actually decreasing in the vicinity of the TC after 
(a) Dependance on cumulus parameterization

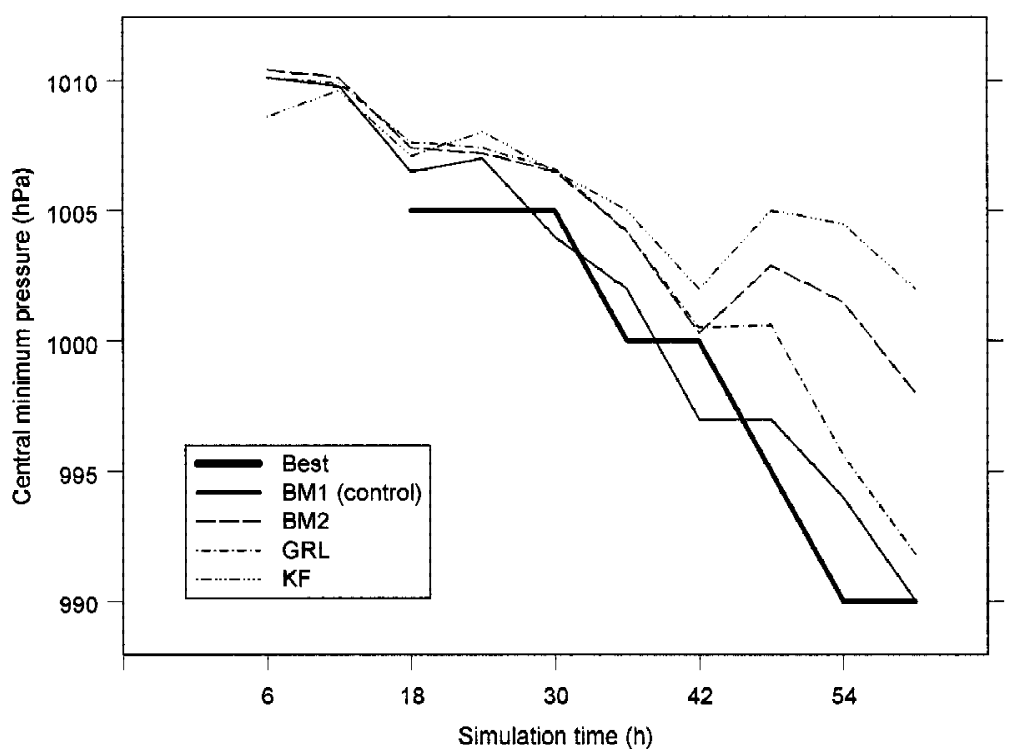

(b) Simulated tracks of Robyn

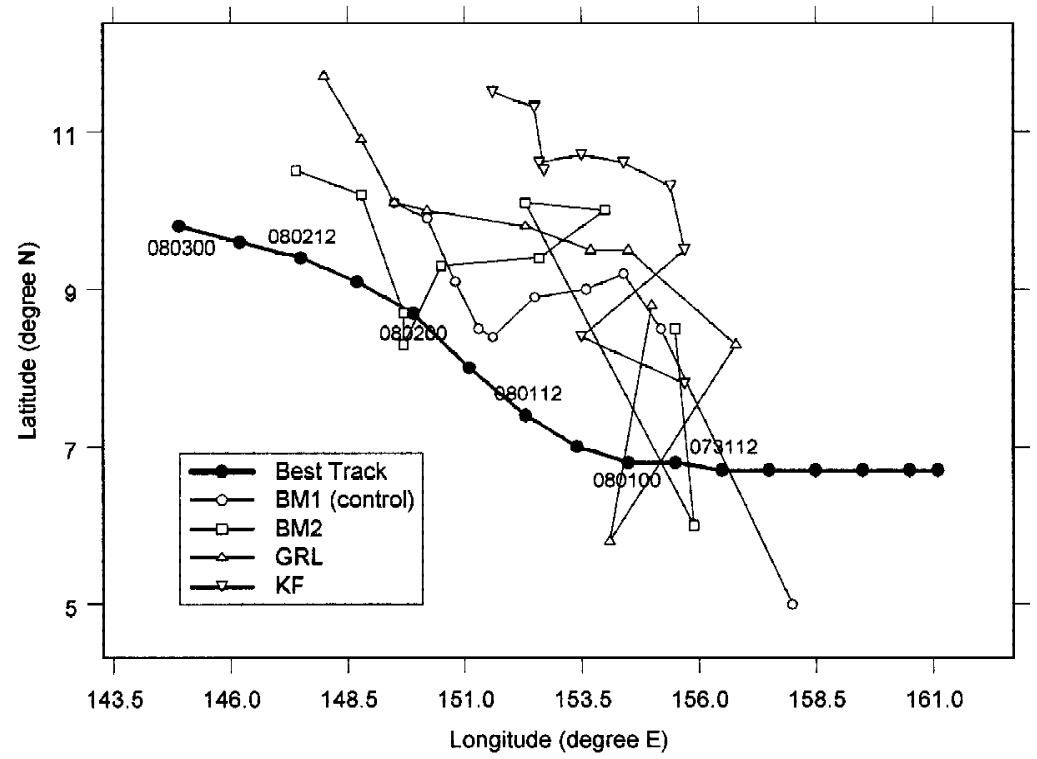

Fig. 15. (a) Time series of central minimum pressure and (b) simulated tracks with 6 hourly positions for experiments BM1, BM2, GRL, and KF. The first position is at 073118 , i.e., $6 \mathrm{~h}$ from the start of the simulation. 
Vortex-centered average 3-hourly accumulated convective rainfall

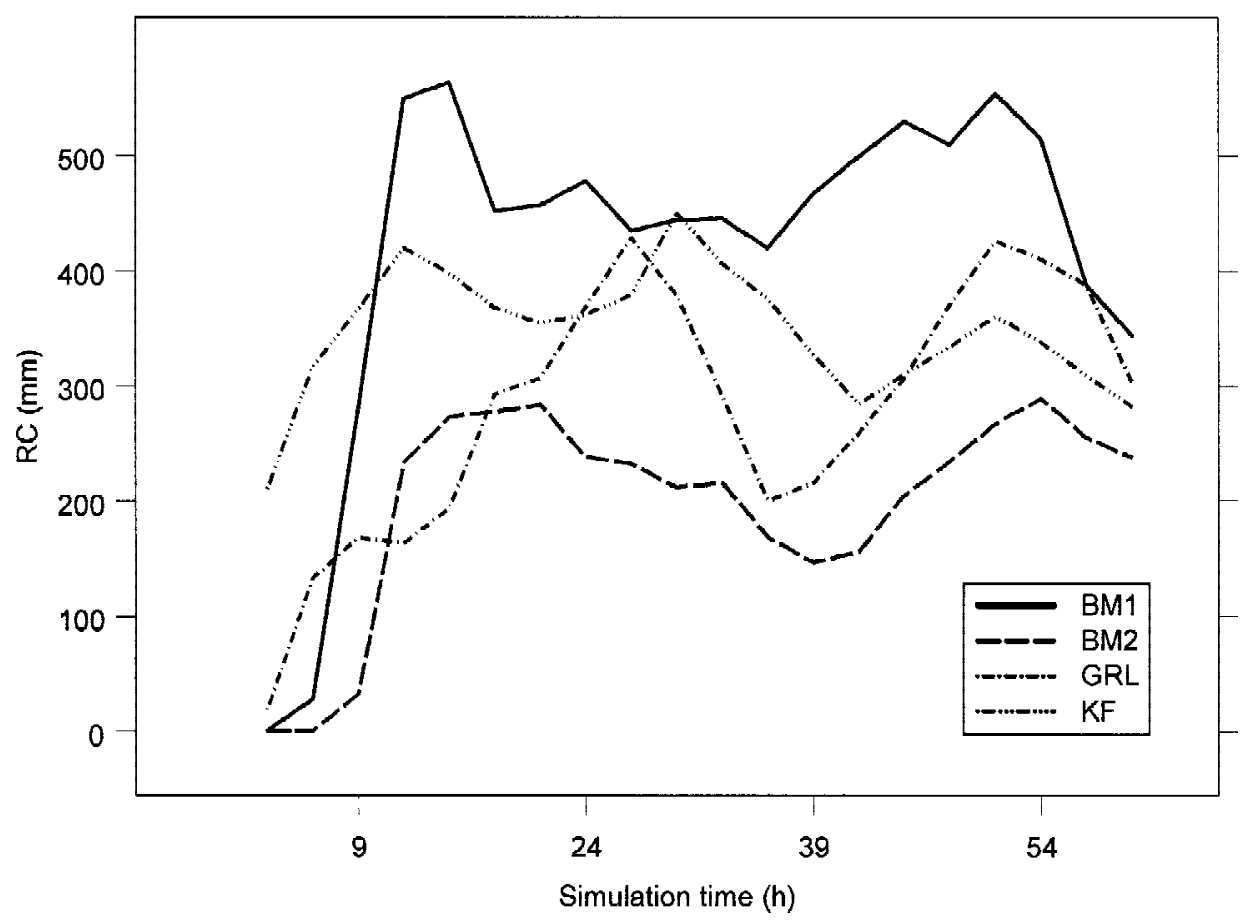

Fig. 16. Time series of vortex-centered average (over an area of $780 \times 1050 \mathrm{~km}$ ) three-hourly accumulated convective rainfall from experiments BM1, BM2, GRL, and KF.

about $30 \mathrm{~h}$ of simulation (Fig. 17). Moreover, the distribution of convective rainfall in KF (Fig. 17) is widely spread and not closely correlated with the development of the three MCSs. The precipitation bands in BM1 and GRL (Fig. 17) look more similar to those in satellite imagery. Because of the specification of a more stable reference profile in BM2, it is expected to generate scattered and smaller amounts of convective rainfall, as is shown in Fig. 17.

Vorticity generation during the TC intensification is also affected by the explicit (nonconvective) precipitation. Consistent with the small intensification of Robyn in the KF experiment, the non-convective rainfall in the KF experiment is lower by at least an order of magnitude than for the other three schemes (not shown). The Grell scheme, which tends to be dominated by explicit precipitation, generates similar amounts as the two Betts-Miller experiments in this case.

The simulations here only partially agree with those by Davis and Bosart (2002), who simulated the formation of Hurricane Diana (1984) using the MM5 model with the same PBL scheme (MRF) as here. The two studies agree relative to the degree of TC intensification and amounts of convective rainfall in the original Betts-Miller scheme compared to the modified 
one, and also the characteristic features of the Grell scheme. The largest difference arises between the BM1 and KF simulations. In Davis and Bosart (2002), the degree of intensification of Hurricane Diana is much larger when using the Kain-Fritsch scheme than using the standard Betts-Miller scheme. Although the Kain-Fritsch convective rain rate is larger than that from Betts-Miller throughout their simulation, the Betts-Miller has the larger total rain rate because it generated more explicit precipitation. Davis and Bosart attributed the differences between the two schemes to the different distributions of precipitation, and eventually to that of the vorticity anomalies relative to the storm center, which have an influence on the axisymmetrization process.

In addition, the differences between Davis and Bosart's results and this study may be due to the grid size. Whereas most other cumulus parameterization schemes simply check for buoyancy by lifting parcels representative of individual layers, the trigger function in the KainFritsch is from the grid-resolved upward motion. With a 9-km resolution nested grid to resolve the vertical velocities, and because the formation of Hurricane Diana was associated with a
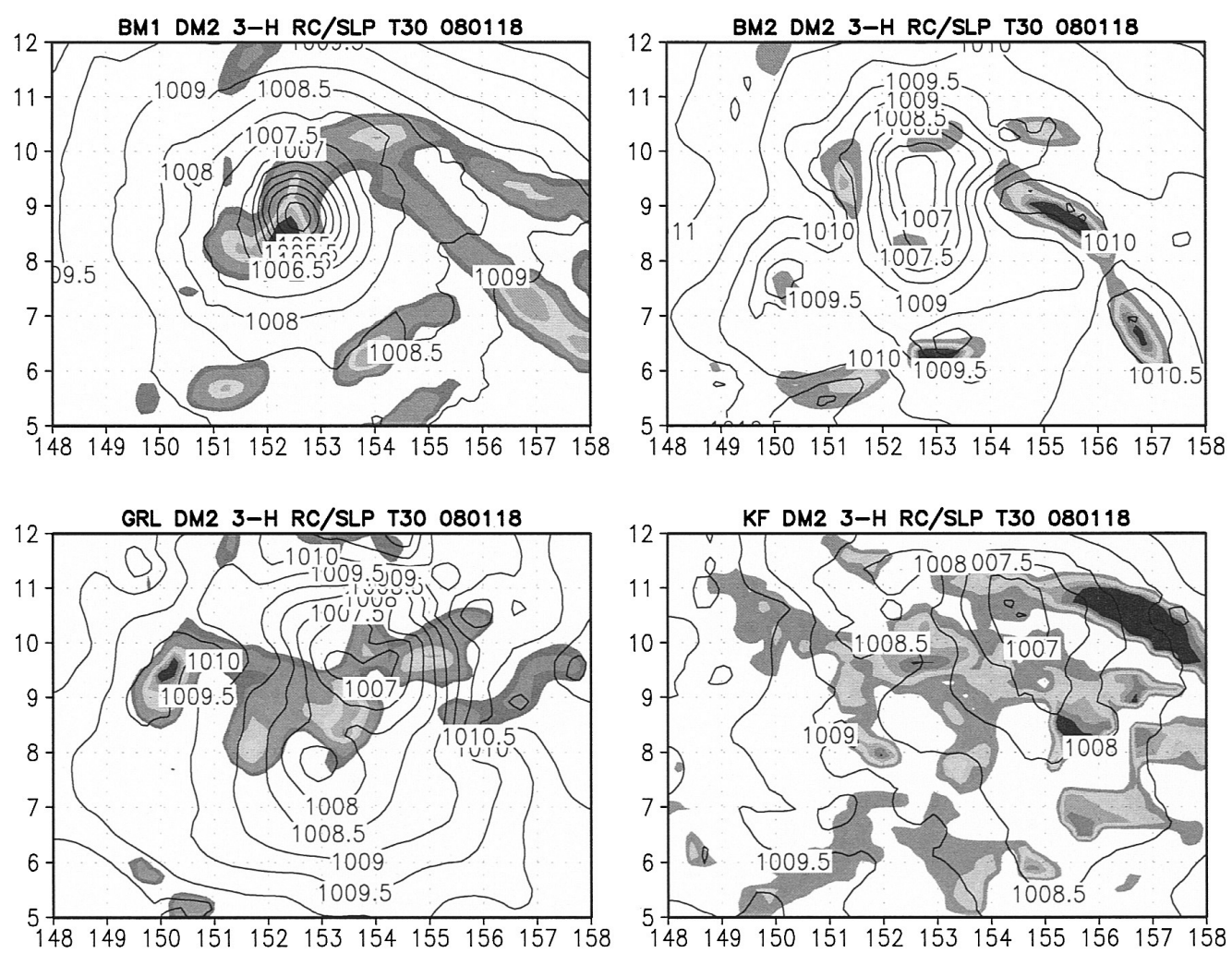

Fig. 17. Mean-sea-level pressure (contours, hPa) and three-hourly accumulated convective rainfall (shaded, levels $>0.5 \mathrm{~cm}$ shown) at 080118 from experiments BM1, BM2, GRL, and KF. 
frontal condition, the Kain-Fritsch scheme in the Davis and Bosart MM5 simulation was highly active. The 27-km resolution inner grid in this study does not resolve the mesoscale updrafts, which may also explain the widely spread convective precipitation in the KF simulation.

\subsection{Sensitivity to PBL Parameterization}

Three other experiments are performed utilizing different PBL parameterizations (Table 1). As mentioned above, the control BM1 experiment uses the PBL scheme as in the NCEP MRF model that has non-local diffusion. In the simulation labeled ETA, the PBL scheme as in the NCEP eta model is used, which applies the Mellor-Yamada prediction of the turbulent kinetic energy (TKE) and has local vertical mixing. The BTS experiment uses the Burk-Thompson PBL scheme, which is also based on the Mellor-Yamada formulas but has a force-restore ground temperature prediction. Another Mellor-Yamada-based scheme tested here is the GaynoSeaman (GSM) scheme. It is expected to be more accurate than the others under saturated conditions due to the use of liquid-water potential temperature as a conserved variable.

Both the intensification and motion of Typhoon Robyn depend little on the choice of PBL scheme for these MM5 simulations (Fig. 18). The deepest intensification occurs with the MRF (control) and ETA schemes, and both agree well with the best-track values. The relative lack of sensitivity in the intensification of Robyn to the PBL scheme, and the similarity of all the simulated tracks to that in the control experiment, suggests at this early stage the surface flux contribution to moisture processes in the model is not a determining factor in the development or non-development of the TC.

An impact from the PBL parameterization did occur away from Typhoon Robyn. A special synoptic feature during the formation of Robyn was the presence of a second monsoon depression at about the same latitude. Whereas convection developed in the eastern monsoon depression that became Robyn, no developing cloud cluster occurred in the western one. Since the inner domain in the MM5 model just covers both depression areas, the western circulation is also examined. In the control simulation (BM1), the MM5 model maintains a weak western low-pressure area until the end of the simulation (Fig. 19, left panels). However, the ETA simulation spins up a cyclone in the western monsoon depression between 080200 and 080300 that has a comparable intensity to Robyn (Fig. 19, right panels). As similar spurious vortices have been observed in the operational models, this simulation was examined further.

Braun and Tao (2000) studied the sensitivity to the PBL parameterizations in MM5 during the intensification of Hurricane Bob (1991) in the Atlantic. Their major conclusion was that differences in the simulated intensification of Bob were largely determined by the surface fluxes rather than by the PBL vertical mixing parameterization. As in the Braun and Tao study, the MRF scheme tends to produce the weakest storm in this study because its excessively deep vertical mixing acts to dry the lower PBL. For example, a similar surface convergence to that in the pre-Robyn stage is simulated in the western monsoon depression at about 080100 (Fig. 20a left panel). Due to the more efficient vertical mixing in the MRF scheme, convection is easily triggered and extended to mid-troposphere in the regions with large surface heat fluxes such as in the circulation near $6^{\circ} \mathrm{N}, 138^{\circ} \mathrm{E}$ (Fig. 20b left panel). Because more moisture is mixed out of the PBL, it resulted in a drier PBL. As the system continues to evolve, 
(a) Dependance on PBL scheme

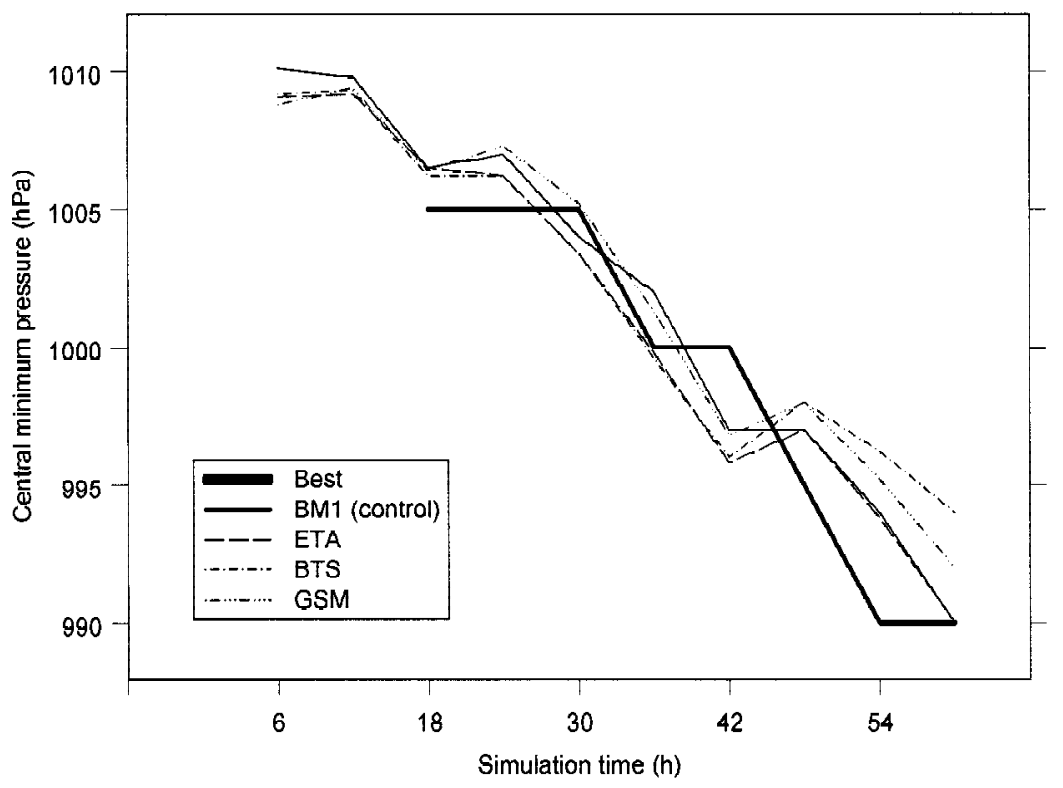

(b) SImulated tracks of Robyn

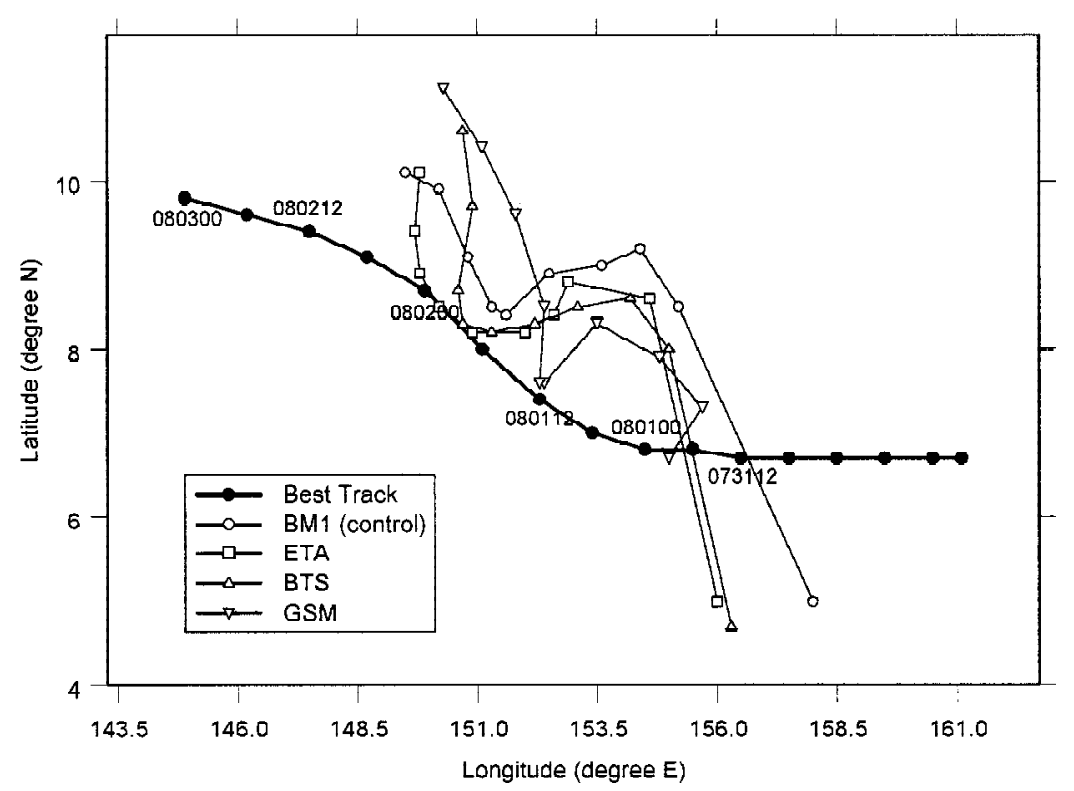

Fig. 18. As in Fig. 15, except for experiments BM1, ETA, BTS, and GSM. 
BM1 DM2 SLP T36 080200

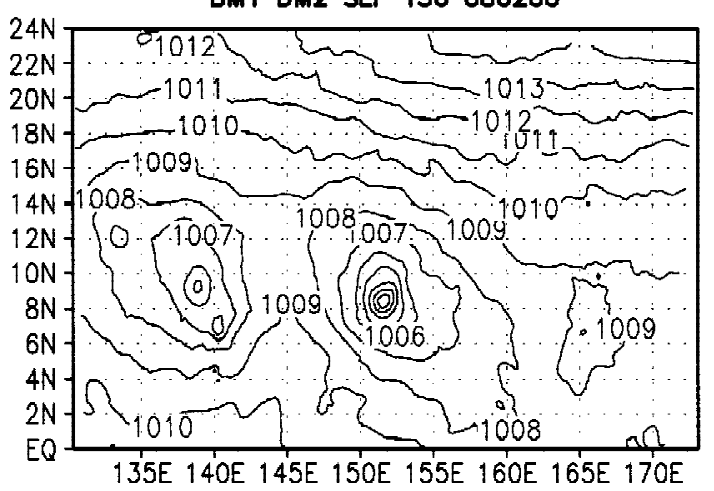

BM1 DM2 SLP T48 080212

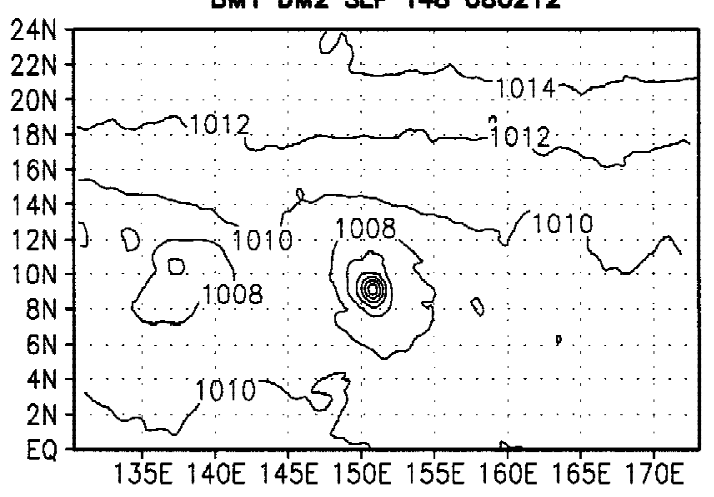

BM1 DM2 SLP T60 080300

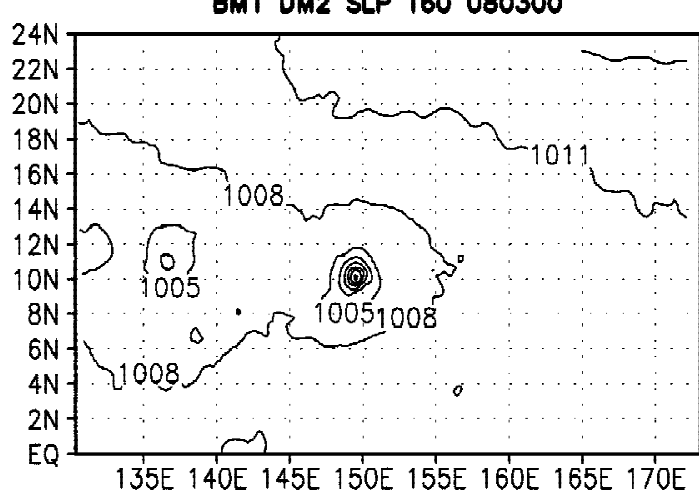

ETA DM2 SLP T36 080200

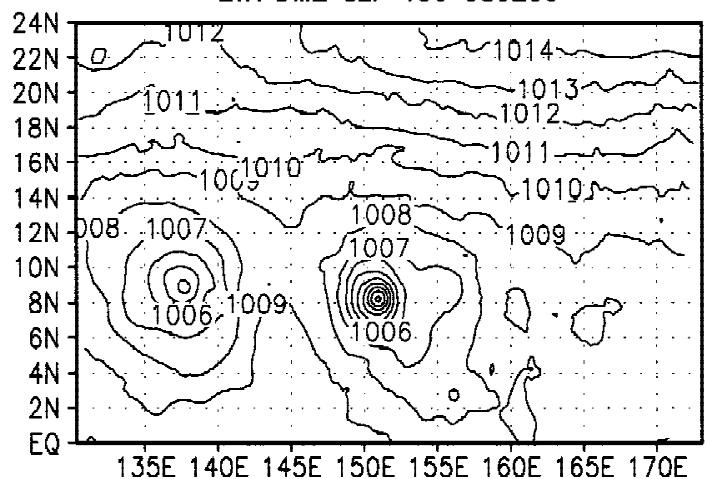

ETA DM2 SLP T48 080212

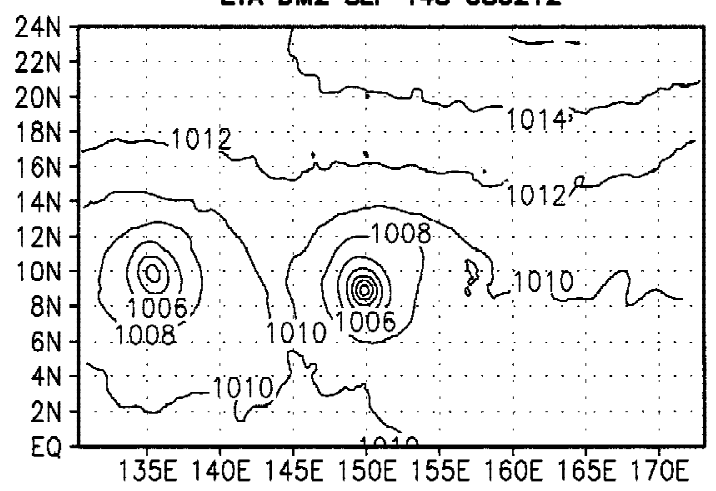

ETA DM2 SLP T60 080300

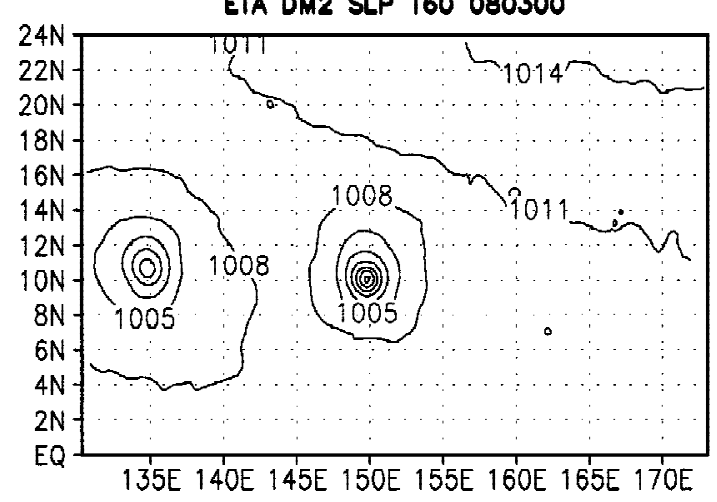

Fig. 19. Mean-sea-level pressures (hPa) in experiments BM1 (left panels) and ETA (right panels) at $36 \mathrm{~h}$ (upper panels), $48 \mathrm{~h}$ (middle panels), and $60 \mathrm{~h}$ (lower panels) of the simulation. 
more convective centers appear (Fig. 20c left panel). However, these centers are widely separated and do not interact. When the low-level vorticity is not enhanced by vertical stretching processes in these convective regions, the BM1 simulation only maintains a moderate western monsoon depression.

With the ETA PBL scheme, a viscous sublayer is assumed to be present only over water (Janjić 1994). Within the viscous sublayer, vertical transports are determined entirely by molecular diffusion. In the turbulent layer above the viscous sublayer, vertical transports are determined by the Mellor-Yamada turbulent fluxes. Due to the presence of this viscous sublayer, convection deeper than the PBL depth is not very active in the ETA simulation. Near where the spurious vortex forms within the western monsoon depression, the strong surface heat fluxes are spread over a larger area than in BM1 (Fig. 20a right panel). However, the cloud water mostly remains below 1 to $2 \mathrm{~km}$ (Fig. 20b right panel), which suggests an overly moist PBL. In general, convective rainfall is light as well as widely spread in the ETA experiment, which is a characteristic also identified by Janjić (1994). This kind of extended precipitation may help spinning up a stronger vortex in ETA compared with the control experiment(Fig. 20c right panel).

The differences between the BM1 and ETA experiments lead to the question: which is a better PBL scheme for TC formation predictions under operational conditions? More case studies would have to be conducted to demonstrate whether the relatively dry PBL from the MRF scheme will consistently result in missed cyclone developments (even though it performed well in this case of Typhoon Robyn). On the other hand, will the viscous-layer effect in the ETA scheme frequently produce spurious vortices? Because either missed or spurious vortices cause problems for forecasters, this may be a great challenge to extending skillful formation predictions a few days ahead.

\subsection{Sensitivity to Explicit Moisture Scheme}

The explicit moisture scheme may be expected to interact with the cumulus parameterization (if one is applied) to determine the development or nondevelopment of the disturbances in the model. One uncertainty with an explicit scheme is how many moisture species need to be incorporated. The simplest scheme is the warm rain model in which only the conversion between the cloud and the rain water mixing ratios is computed. One of the most sophisticated schemes from Reisner et al. (1998) is adopted in the control experiment BM1. The Reisner scheme includes the snow, ice and graupel phases, so that their number concentrations must be predicted. Some less complicated (and hence less computationally demanding) schemes are tested here to see if they are able to reproduce similar results as the Reisner scheme. In experiment SIMICE, a much simpler scheme by Dudhia (1989) is used in which only the cloud water and rain water/snow mixing ratio are predicted separately. Experiment MIX utilizes the mixedphase model also described in Reisner (1998), but with the number concentration of graupel and cloud ice fixed rather than predicted. The Schultz (1995) scheme, which also treats ice, snow and graupel mixing ratios, is also used because it offers better computational efficiency. All of these explicit moisture sensitivity experiments use the same Betts-Miller cumulus parameterization. 
(a)
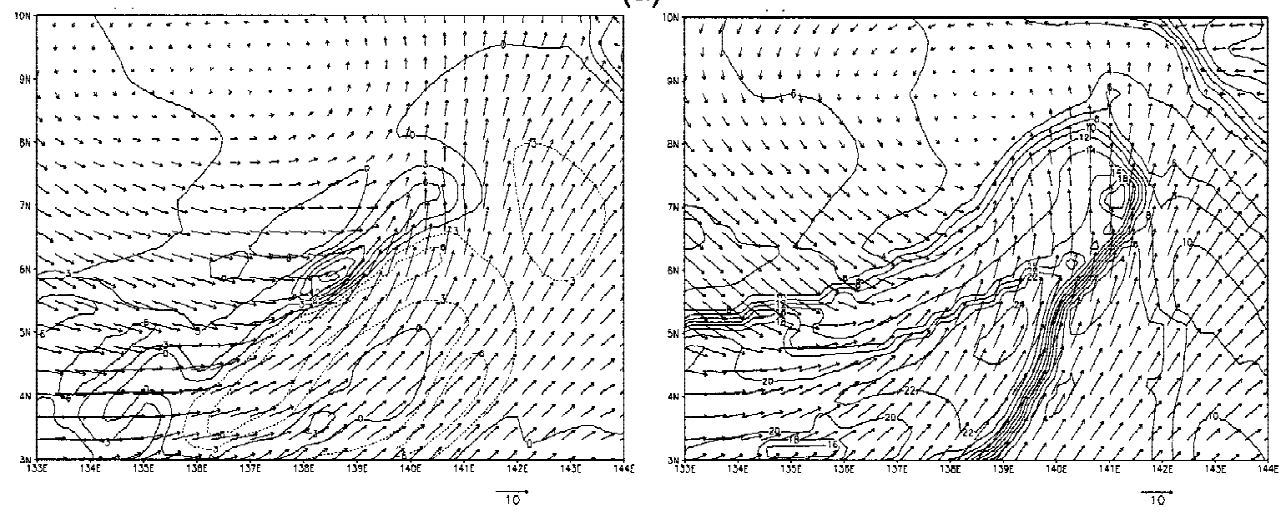

(b)
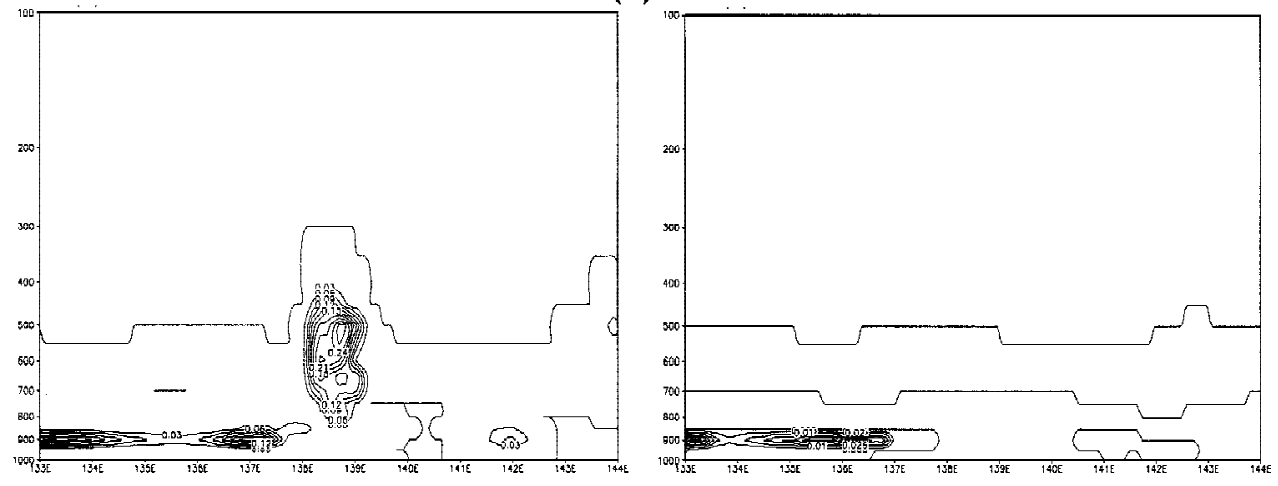

(c)
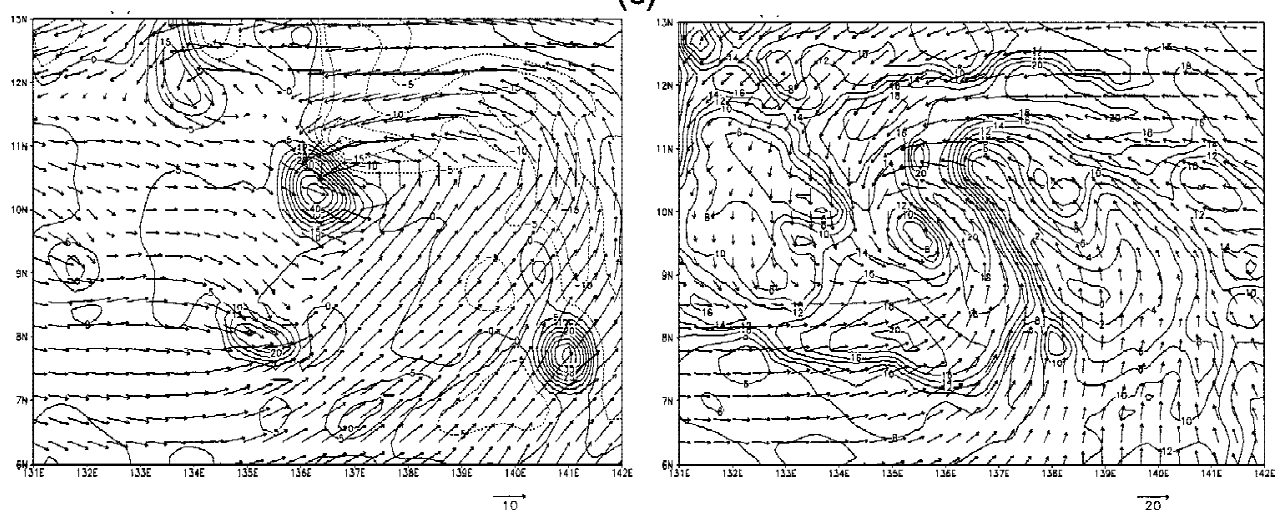

Fig. 20. Surface winds and sensible heat fluxes (unit: $\mathrm{Wm}^{-2}$ ) from experiment BM1 (left panels) and ETA( right panels) at (a) 080100 and (c) 080212. (b) Vertical east-west cross section at $6^{\circ} \mathrm{N}$ of cloud water mixing ratio (unit: $\mathrm{g} \mathrm{kg}^{-1}$ ) at 080100 for BM1 and ETA. 
The simulation of the intensification of Robyn is indeed sensitive to the explicit moisture scheme (Fig. 21a), although the sensitivity is smaller than that for cumulus parameterization. The SIMICE, MIX, and Schultz experiments all underestimate Robyn's intensity, with large deviations from the control simulation beginning about $36 \mathrm{~h}$. Whereas in the SIMICE and MIX experiments the TC almost stops intensifying after $42 \mathrm{~h}$, the Schultz scheme keeps deepening the vortex so that the 60 -h error in minimum surface pressure is only $4 \mathrm{hPa}$. As in the PBL sensitivity tests, the tracks from these experiments are similar (Fig. 21b). The vortices in SIMICE and MIX move farther to the west probably because they are weaker than those in BM1 and Schultz, and their structure is more easily modified by the environmental flow in the monsoon depression. These four experiments indicate that predicting the different moisture species in the model indeed is essential to simulating correctly the formation and early intensification stage of this TC.

These four explicit moisture scheme experiments produce relatively similar non-convective precipitation amounts (Fig. 22). For example, the inner nest average non-convective rainfall has a peak at about $24 \mathrm{~h}$ when the convection develops in the northern part of the monsoon depression. Although the precipitation is moderate during the next 15 to $18 \mathrm{~h}$, the system keeps intensifying. The different intensifications among the experiments are due to the impact of the explicit moisture calculation on the cumulus parameterization. At 080206, the convective rainfalls from the experiments have huge contrasts (Fig. 23). In the control experiment BM1, the cloud systems have already developed into a column structure (not shown). The other three experiments have only individual cloud line structures that do not have a wellorganized cyclonic curvature. In particular, the similarity of the Reisner algorithms in BM1 and MIX (predicted ice/snow/graupel concentrations versus fixed) makes this quite a dramatic contrast.

\section{SUMMARY AND DISCUSSION}

The PSU-NCAR mesoscale model MM5 with an 81-km resolution coarse grid and 27-km resolution inner grid is used to simulate the formation of Typhoon Robyn (1993) in the western North Pacific. This study examines the roles of mesoscale convective system (MCS) during Robyn's formation and also focuses on the sensitivity of the simulations of Robyn's intensification to the choice of cumulus parameterization, PBL parameterization, and explicit moisture scheme in the model. Among the experiments, the control experiment (BM1) with the Betts-Miller cumulus parameterization, PBL treatment as in the NCEP MRF model, and Reisner explicit moisture scheme with predicted number concentrations of snow, ice, and graupel has the closest intensification profile to the best track.

Other comparisons of the control experiment with available observations collected during the Tropical Cyclone Motion (TCM-93) experiment are made to further validate the model. In particular, observations from the aircraft observation period (AOP-3A) that occurred just before the first warning of Robyn with NOGAPS large-scale fields as background, and then compared to the MM5 simulations. The control simulation is generally able to reproduce the successive MCS systems occurring during the formation of Robyn, although there are spatial 
(a) Dependance on explicit moisture scheme

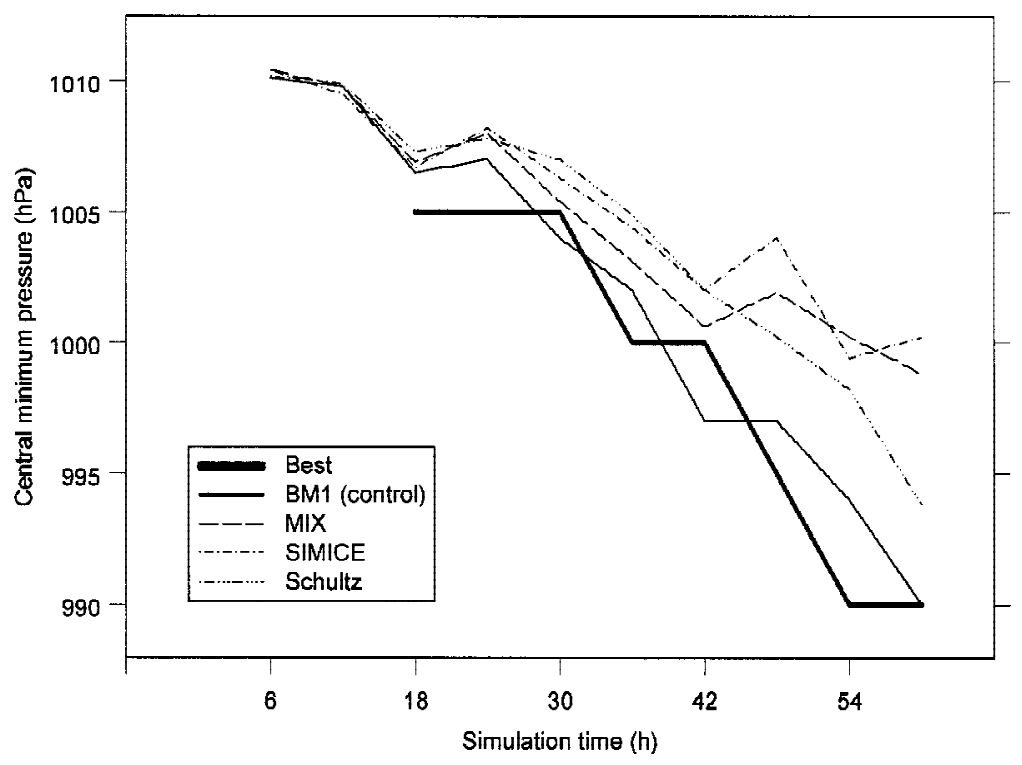

(b) SImulated tracks of Robyn

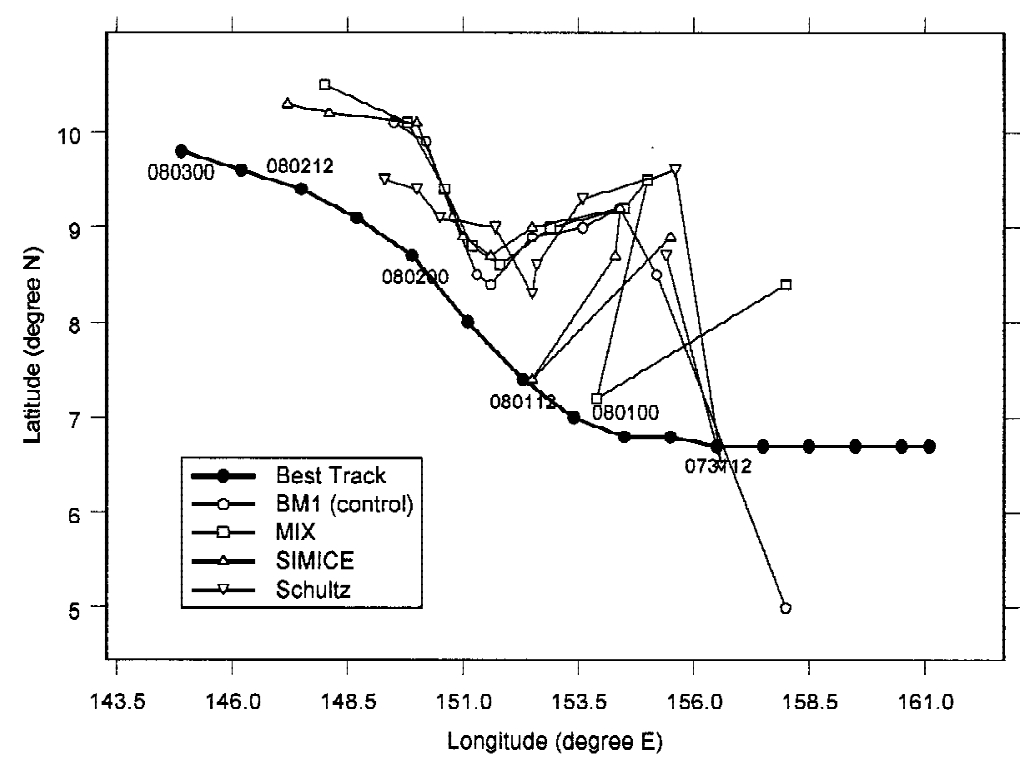

Fig. 21. As in Fig. 15, except for experiments BM1, MIX, SIMICE, and Schultz. 


\section{Domain-averaged 3-hourly accumulated non-convective rainfall}

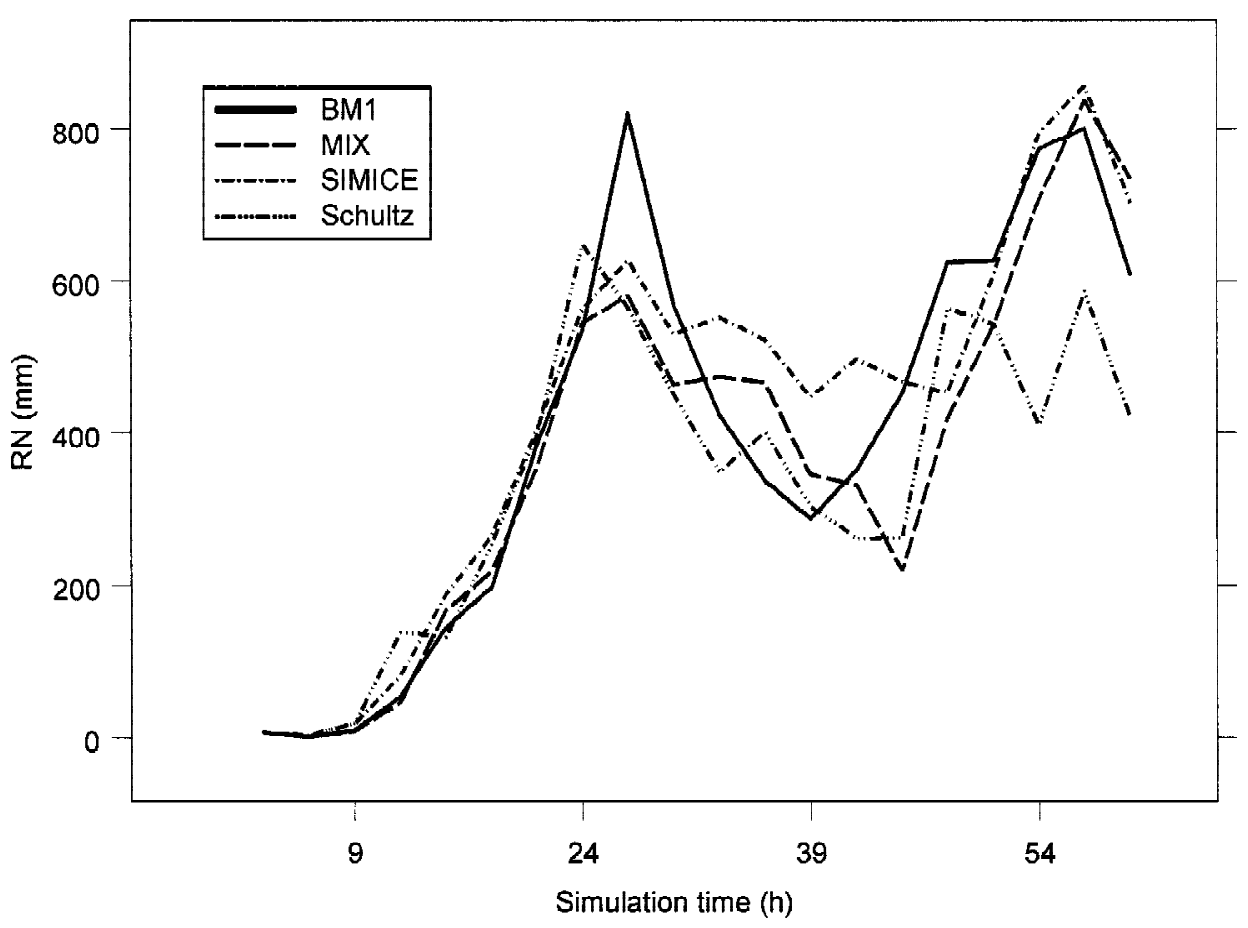

Fig. 22. Time series of inner domain-averaged three-hourly accumulated nonconvective rainfall from experiments BM1, MIX, SIMICE, and Schultz.

and temporal deviations from those revealed in satellite imagery. However, the simulated fields do include more mesoscale features than the analyzed fields such as some mesoscale circulations associated with the convective centers. While the analyzed fields are only rough approximations of the actual mesoscale circulations due to the spatial separation of the dropwindonde observations (most of them are about $100 \mathrm{~km}$ apart), the validation of the control simulation primarily from satellite evidence of MCSs cannot be said to be conclusive. Simulations of more recent TC formation cases, utilizing high-resolution products such as satellite-derived winds and in situ aircraft observations, are required to enable more detailed grid-scale validations.

The system development during the formation of Robyn as revealed in the MM5 simulations is shown to be consistent with Zehr's two-stage development conceptual model. However, a hypothesis is proposed that the surge necessary for a second major convection does not necessarily come from the large-scale southwesterlies, but can also be from the previous local interaction of MCSs embedded in the monsoon depression.

Sensitivity experiments with different cumulus parameterizations demonstrate that this 

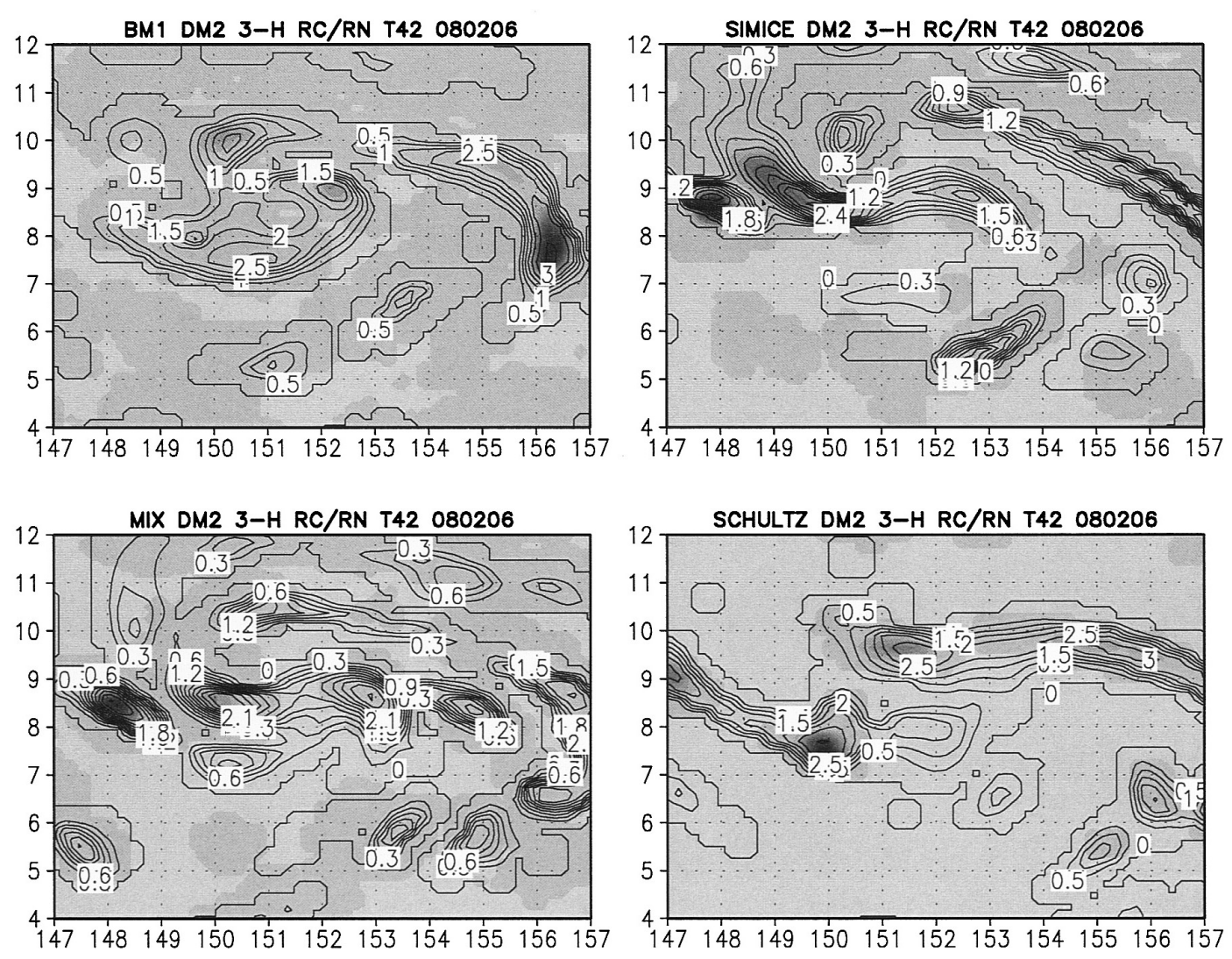

Fig. 23. Three-hourly accumulated convective (contour) and non-convective (shaded) rainfall at 080206 from experiments BM1, MIX, SIMICE, and Schultz (unit: $\mathrm{cm}$ ).

choice largely determines the degree of intensification of Robyn. Experiment BM2 with a more tropical reference profile (i.e., less unstable) in the Betts-Miller scheme, and the simulations with the Kain-Fritsch (KF) scheme and Grell (GRL) scheme all underestimate the intensity of Robyn throughout the model integration. Whereas most of the results may be interpreted from the inherent characteristics of each parameterization (e.g., the amount of convective rainfall each scheme produces), the relative outcomes from the BM1 and KF simulations do not agree with the Davis and Bosart (2002) study in which the formation of Hurricane Diana (1984) was simulated using the same model. In the Davis and Bosart's simulations, the Kain-Fritsch scheme generates a deeper intensification of Hurricane Diana than does the standard Betts-Miller scheme. In this study, the Kain-Fritsch scheme simulates a widely spread convection pattern that leads to the non-development of the associated cloud clusters. It is 
believed that these differences may be due to the lower (27 km versus $9 \mathrm{~km}$ ) model grid resolution in this study, and the different synoptic situation associated with the two formation cases.

In addition to the NCEP MRF model, the PBL scheme from the NCEP eta model (ETA), the Burk-Thompson scheme (BTS), and the Gayno-Seaman scheme (GSM) are tested. The impacts on the intensification and track of Typhoon Robyn due to changes in the PBL treatment are found to be small. However, a peculiar feature in experiment ETA is that a spurious vortex is spun up in the monsoon depression west of Robyn. Comparisons of the predicted convection and cloud water in the PBL from these two experiments reveal that the PBL in the MRF scheme is relatively dry (as found by Braun and Tao 2000) due to its active vertical mixing. The eta PBL is found to be overly moist with extended precipitation probably due to the viscous sublayer in the scheme, which evidently leads to the spurious vortex generation.

Although the impact is smaller than that of the cumulus parameterization, using different explicit moisture schemes indeed affects the simulation of the intensification of Typhoon Robyn through the interaction of the explicit moisture with the Betts-Miller cumulus parameterization. The three explicit schemes tested [simple ice (SIMICE) calculation, the mixed-phase model (MIX), and the Schultz scheme] all underestimate the intensity of Robyn compared to the complex and computationally demanding Reisner scheme. This suggests a decision between computational efficiency and accuracy may have to be made because the other three schemes include fewer predicted moisture species to reduce the computational burden. Among the experiments, the Schultz scheme may be an alternative to the control Reisner scheme due to its efficient computations, since it is still able to reproduce a reasonable intensification profile of Robyn. All of the explicit moisture calculations generate similar amounts of non-convective rainfall. However, major deviations in convective rainfall among these experiments arise due to modified convective cloud patterns by the explicit moisture variables.

Simulations with a configuration similar to the control experiment in this study and with higher resolution versions of the model will be presented in a later report along with more detailed diagnostics through budget analysis. Nevertheless, the moderate-resolution experiments here may have relevance to operational forecasting in that prediction of TC formation time and location might be improved by using such a mesoscale model. Addition of a mesoscale component in a hierarchical prediction system of TC formation would extend downscale the influences of global and synoptic on space and time scales. On the climatic time scales, TC activity may be modulated by climatic signals such as the El Niño Southern Oscillation and other planetary-scale oscillations. On the intraseasonal time scale, the Madden-Julian oscillations may have to be well captured to understand the variability of activity and inactivity within a season. For medium-range numerical models, it might still be questionable if they simulate the intraseasonal oscillations well enough for this purpose. Once enhanced convective systems develop in a circulation, it usually takes days for it to develop into a tropical cyclone. It is exactly the skill of predictions on this time scale that is most important to releasing effective early warnings and therefore needs much improvement. A consistently skillful mesoscale model might be expected to fit into this downscaling approach, and efforts in developing and testing such a model through many case studies are seen to have immediate importance. 
Acknowledgments Many of the model diagnostics in this study were performed using a Grid Analysis and Display System (GrADS) utility program contributed by George Bryan of the Pennsylvania State University to the NCAR MM5 mesoscale modeling community. The comments from the two anonymous reviewers are much appreciated. This research is supported by the U. S. Office of Naval Research Marine Meteorology section.

\section{REFERENCES}

ATCR, 1993: Annual tropical cyclone report. U.S. Naval Pacific Meteorology and Oceanography Center/Joint Typhoon Warning Center, Guam, Mariana Islands, 234 pp.

Bister, M., and K. A. Emanuel, 1997: The genesis of hurricane Guillermo: TEXMEX analyses and a modeling study. Mon. Wea. Rev., 125, 2662-2682.

Braun, S. A., and W. K. Tao, 2000: Sensitivity of high-resolution simulations of Hurricane Bob (1991) to planetary boundary layer parameterizations. Mon. Wea. Rev., 128, 3941-3961.

Chen, S. S., and W. M. Frank, 1993: A numerical study of the genesis of extratropical convective mesovortices. Part I: Evolution and dynamics. J. Atmos. Sci., 50, 2401-2426.

Cheung, K. K. W., 2004: Large-scale environmental parameters associated with tropical cyclone formations in the western North Pacific. J. Climate, 17, 466-484.

Cheung, K. K. W., and R. L. Elsberry, 2002: Tropical cyclone formations over the western North Pacific in the Navy Operational Global Atmospheric Prediction System forecasts. Wea. Forecasting, 17, 800-820.

Davis, C. A., and L. F. Bosart: 2001 Numerical simulations of the genesis of Hurricane Diana (1984). Part I: Control simulation. Mon. Wea. Rev., 129, 1859-1881.

Davis, C. A., and L. F. Bosart: 2002 Numerical simulations of the genesis of Hurricane Diana (1984). Part II: Sensitivity of track and intensity prediction. Mon. Wea. Rev., 130, 11001124.

Dudhia, J., 1989: Numerical study of convection observed during the winter monsoon experiment using a mesoscale two-dimensional model. J. Atmos. Sci., 46, 3077-3107.

Gray, W. M., 1968: Global view of the origin of tropical disturbances and storms. Mon. Wea. Rev., 96, 669-700.

Harr, P. A., R. L. Elsberry, E. A. Ritchie, H. E. Willoughby, M. A. Boothe, L. E. Carr III, and R. A. Jeffries, 1993: Tropical Cyclone Motion (TCM-93) Field Experiment Summary. Report NPS-MR-93-004, Naval Postgraduate School, Monterey, California, 120 pp.

Harr, P. A., R. L. Elsberry, and J. C. L. Chan, 1996a: Transformation of a large monsoon depression to a tropical storm during TCM-93. Mon. Wea. Rev., 124, 2625-2643.

Harr, P. A., M. S. Kalafsky, and R. L. Elsberry, 1996b: Environmental conditions prior to formation of a midget tropical cyclone during TCM-93. Mon. Wea.Rev., 124, 16931710.

Hendricks, E. A., M. T. Montgomery, and C. A. Davis, 2004: The role of "vortical" hot towers in the formation of tropical cyclone Diana (1984). J. Atmos. Sci., 61, 12091232. 
Janjić, Z. I., 1994: The step-mountain eta coordinate model: Further developments of the convection, viscous sublayer, and turbulence closure schemes. Mon. Wea. Rev., 122, 927-945.

Kuo, H. C., J. H. Chen, R. T. Williams, and C. P. Chang, 2001: Rossby waves in zonally opposing mean flow: behavior in Northwest Pacific summer monsoon. J. Atmos. Sci., 58, 1035-1050.

McBride, J. L., 1981: Observational analysis of tropical cyclone formation. Part III: Budget analysis. J. Atmos. Sci., 38, 1152-1166.

McBride, J. L., and R. Zehr, 1981: Observational analysis of tropical cyclone formation. Part II: Comparison of non-developing versus developing systems. J. Atmos. Sci., 38, 11321151.

Reisner, J. R., M. Rasmussen, and R. T. Bruintjes, 1998: Explicit forecasting of supercooled liquid water in winter storms using the MM5 mesoscale model. Quart. J. Roy. Meteor. Soc., 124, 1071-1107.

Reynolds, R. W., and T. M. Smith, 1994: Improved global sea surface temperature analyses using optimum interpolation. J. Climate, 7, 929-948.

Ritchie, E. A., J. Simpson, W. T. Liu, J. Halverson, C. S. Velden, K. F. Brueske, and H. Pierce, 2003: Present day satellite technology for hurricane research. In: Simpson, R., (Ed.), Hurricane! Coping with Disaster. Am. Geophy. Union, 360 pp.

Rogers, R. F., and J. M. Fritsch, 2001: Surface cyclogenesis from convectively driven amplication of midlevel mesoscale convective vortices. Mon. Wea. Rev., 129, 605-637.

Schultz, P., 1995: An explicit cloud physics parameterization for operational numerical weather prediction. Mon. Wea. Rev., 123, 3331-3343.

Zehr, R. M., 1992: Tropical cyclogenesis in the western North Pacific. NOAA Tech. Rep. NESDIS 61. U. S. Department of Commerce, Washington, DC 20233, 181 pp. 